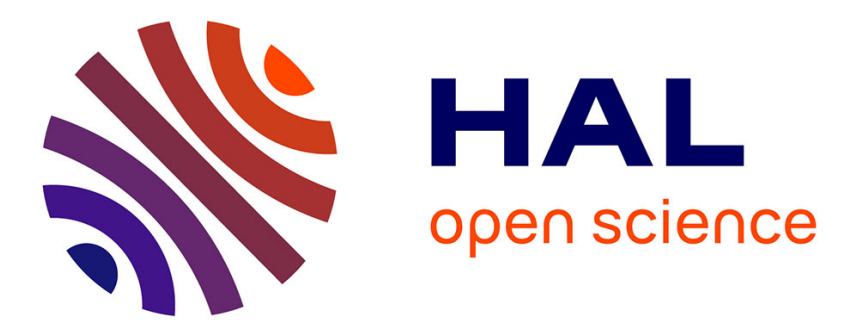

\title{
The steady state configurational distribution diffusion equation of the standard FENE dumbbell polymer model: existence and uniqueness of solutions for arbitrary velocity gradients
}

\author{
Ionel Sorin Ciuperca, Liviu Iulian Palade
}

\section{To cite this version:}

Ionel Sorin Ciuperca, Liviu Iulian Palade. The steady state configurational distribution diffusion equation of the standard FENE dumbbell polymer model: existence and uniqueness of solutions for arbitrary velocity gradients. 2009. hal-00363084

\author{
HAL Id: hal-00363084 \\ https://hal.science/hal-00363084
}

Preprint submitted on 20 Feb 2009

HAL is a multi-disciplinary open access archive for the deposit and dissemination of scientific research documents, whether they are published or not. The documents may come from teaching and research institutions in France or abroad, or from public or private research centers.
L'archive ouverte pluridisciplinaire HAL, est destinée au dépôt et à la diffusion de documents scientifiques de niveau recherche, publiés ou non, émanant des établissements d'enseignement et de recherche français ou étrangers, des laboratoires publics ou privés. 


\title{
The steady state configurational distribution diffusion
}

\section{equation of the standard FENE dumbbell polymer model:}

\author{
existence and uniqueness of solutions for arbitrary \\ velocity gradients.
}

Ionel Sorin Ciuperca ${ }^{1}$ and Liviu Iulian Palade ${ }^{2}$

February 20, 2009

Université de Lyon, CNRS

${ }^{1}$ Université Lyon 1, Institut Camille Jordan UMR5208, Bât Braconnier, 43 Boulevard du 11

Novembre 1918, F-69622, Villeurbanne, France.

${ }^{2}$ INSA-Lyon, Institut Camille Jordan UMR5208 \& Pôle de Mathématiques, Bât. Leonard de Vinci No. 401, 21 Avenue Jean Capelle, F-69621, Villeurbanne, France.

\begin{abstract}
The configurational distribution function, solution of an evolution (diffusion) equation of the Fokker-Planck-Smoluchowski type, is (at least part of) the corner stone of polymer dynamics: it is the key to calculating the stress tensor components. This can be reckoned from [1], where a wealth of calculation details is presented regarding various polymer chain models and their ability to accurately predict viscoelastic flows. One of the simplest polymer chain idealization is the Bird and Warner's model of finitely extensible nonlinear elastic (FENE) chains. In this work we offer a proof that the steady state
\end{abstract}


configurational distribution equation has unique solutions irrespective of the (outer) flow velocity gradients (i.e. for both slow and fast flows).

Keywords: FENE dumbbell chains; Fokker-Planck-Smoluchowski equation; existence and uniqueness of solutions; slow and fast viscoelastic flows; Krein-Rutman theorems;

\section{Introduction}

The viscoelastic flow behavior of polymeric liquids is strongly influenced by the complexity of various inter and intra molecular interactions. At microscopic level, long chain entanglements are a consequence of chain connectivity and backbone uncrossability due to intermolecular repulsive exclusive volume forces. Macromolecules diffusion (and conformational relaxation) is slowed down due to hydrodynamic drag and Brownian forces.

Bird, Curtiss, Armstrong and Hassager, together with their collaborators (see [1]] and references cited therein), enriched significantly Kirkwood's early ideas [2] and produced a general kinetical theoretical framework for both diluted and concentrated polymeric systems. Here, the macromolecules are modeled as freely jointed bead-rod or bead-spring chains. One of the simplest version of this chain model is the (now popular) Bird - Warner's elastic dumbbell chain, that consists of two beads connected by a Finitely Extensible Nonlinear Elastic - aka FENE spring. The salient features of this model, of relevance to this work, are briefly reviewed below, for sake of clarity.

Let $\tilde{\mathbf{x}} \in \mathbb{R}^{n}, n=2,3$, denote the (microscopic) dumbbell connector vector, $\mathbf{y} \in \mathbb{R}^{n}$ the (macroscopic) Eulerian position vector. In the absence of inertia and of external forces, the balance of hydrodynamic, Brownian and intermolecular forces results in the so-called FokkerPlanck-Smoluchowski with Dirichlet boundary condition for the configurational function 
$\tilde{\psi}(t, \mathbf{y}, \tilde{\mathbf{x}})$ diffusion equation. In dimensionless form it looks:

$$
\begin{aligned}
& \frac{\partial \tilde{\psi}}{\partial t}+\mathbf{u} \cdot \nabla_{\mathbf{y}} \tilde{\psi}=\nabla_{\tilde{\mathbf{x}}} \cdot\left[-\boldsymbol{\theta} \tilde{\mathbf{x}} \tilde{\psi}+\frac{1}{2 \mathrm{De}} \nabla_{\tilde{\mathbf{x}}} \tilde{\psi}+\frac{1}{2 \mathrm{De}} \mathbf{F}^{(c)}(\tilde{\mathbf{x}}) \tilde{\psi}\right], \text { over } B(0, \tilde{\delta}) \\
& \left.\tilde{\psi}\right|_{\partial B(0, \tilde{\delta})}=0
\end{aligned}
$$

In the above equation, $B(0, \tilde{\delta})$ is the open ball of radius $\tilde{\delta}$ centered at 0 , De is the Deborah number and $\boldsymbol{\theta}=\left(\nabla_{\mathbf{y}} \mathbf{u}\right)^{T}$ is a tensor which represents the (macroscopic) velocity gradient; the corresponding term accounts for the flow type. The second term in the rhs represents the statistically averaged Brownian force due to thermal fluctuations in the liquid. The last term, $\mathbf{F}^{(c)}$, is the elastic force that accounts for the dumbbell's elastic response to strain input, for which Warner [3] proposed the following expression (valid for $\|\mathbf{x}\|<\tilde{\delta}$, with $\tilde{\delta}$ a polymer depending parameter):

$$
\mathbf{F}^{(c)}(\tilde{\mathbf{x}})=\frac{\tilde{\mathbf{x}}}{1-(\|\tilde{\mathbf{x}}\| / \tilde{\delta})^{2}}
$$

The above is commonly called the FENE force. Now, as an aside, the model is quite flexible in that it may sustain other types of elastic forces: e.g. Peterlin's force (actually a linearized version of eq.(2) ) usually referred to as FENE-P (see 近, 5]):

$$
\mathbf{F}^{(c)}(\tilde{\mathbf{x}})=\frac{\tilde{\mathbf{x}}}{1-<\|\tilde{\mathbf{x}}\|^{2}>/ \tilde{\delta}^{2}}=\frac{\tilde{\mathbf{x}}}{1-<\operatorname{tr}(\tilde{\mathbf{x}} \otimes \tilde{\mathbf{x}})>/ \tilde{\delta}^{2}}
$$

Asymptotic solutions to the diffusion equation are known for some steady state flows: see [1] (for concise presentations see [6, 7, 8, 9, 10]). They were obtained through series expansions about the (known) equilibrium function $\tilde{\psi}_{e q}(\tilde{\mathbf{x}})$.

Next, let $(t, \mathbf{y}) \in \mathbb{R}_{+} \times\left(Q \subset \mathbb{R}^{n}\right)$. The momentum balance equation reads (see [1]):

$$
\begin{aligned}
& \frac{\partial \mathbf{u}}{\partial t}+\left(\mathbf{u} \cdot \nabla_{\mathbf{y}}\right) \mathbf{u}=\nu \Delta u-\nabla_{\mathbf{y}} p+\nabla_{\mathbf{y}} \cdot \mathbf{S}, \quad \text { over } \mathbb{R}_{+} \times Q \\
& \nabla_{\mathbf{y}} \cdot \mathbf{u}=0, \quad \text { over } \mathbb{R}_{+} \times Q
\end{aligned}
$$

where $\nu>0, \mathbf{u}=\mathbf{u}(t, \mathbf{y}) \in \mathbb{R}^{n}, p=p(t, \mathbf{y}) \in \mathbb{R} . \mathbf{S}(t, \mathbf{y}) \in \operatorname{Sym}(\mathbb{R})$ is the symmetric extra stress tensor given by ([1]): 


$$
\mathbf{S}(t, \mathbf{y})=\mu\left[\int_{B(0, \tilde{\delta})} \tilde{\mathbf{x}} \otimes \mathbf{F}(\tilde{\mathbf{x}}) \tilde{\psi}(t, \mathbf{y}, \tilde{\mathbf{x}}) \mathrm{d} \tilde{\mathbf{x}}-\int_{B(0, \tilde{\delta})} \tilde{\psi}(t, \mathbf{y}, \tilde{\mathbf{x}}) \mathrm{d} \tilde{\mathbf{x}} \mathbf{I}\right]
$$

where $\mu>0$ is a fluid related parameter (actually a given constant).

One observes that whenever the velocity gradient is such that $\partial u_{i} / \partial y_{j}=a_{i j}=$ constant, $\sum_{i} a_{i i}=0$, and $\tilde{\psi}$ is a solution of (1a)-(1b), then $\mathbf{S}$ defined in equation (5) is always independent of $\mathbf{y}$, hence $\nabla_{\mathbf{y}} \cdot \mathbf{S}=0$. In such a situation there exist $\mathbf{u}$ and $p$ so that (4a)-(4b) are solved. That this is indeed the case may be inferred from the following. Using Einstein's summation convention over dummy indices, $u_{i}=a_{i j} y_{j}+c$, therefore $\frac{\partial u_{i}}{\partial y_{k}} u_{k}=a_{i k}\left[a_{k j} y_{j}+c\right]=a_{i k} a_{k j} y_{j}+d_{i}$, $d_{i}=c \sum_{i, k} a_{i k}$. Hence $\left[\nabla_{\mathbf{y}} \cdot(\mathbf{u} \otimes \mathbf{u})\right]_{i}=a_{i k} a_{k j} y_{j}+d_{i}=\alpha_{i j} y_{j}+d_{i}$. Therefore $\nabla_{\mathbf{y}} \cdot(\mathbf{u} \otimes \mathbf{u})$ may be expressed as $\nabla_{\mathbf{y}} \cdot(\mathbf{u} \otimes \mathbf{u})=-\nabla_{\mathbf{y}} p$, where $p=-(1 / 2) \alpha_{i j} y_{i} y_{j}-d_{i} y_{i}$, since the matrix of entries $\alpha_{i j}$ is symmetric. We conclude that for any traceless matrix $\mathbf{A}$ whose entries $a_{i j}$ are constants, and for a steady state, homogeneous flow solution $\tilde{\psi}(\tilde{\mathbf{x}})$ - i.e. independent of $t$ and $\mathbf{y}$ - to equations (1a)-(1b), there exists a steady state solution to (4a)-(4b) given by:

$$
\begin{aligned}
u_{i}(\tilde{\mathbf{x}}) & =a_{i j} y_{j}+c \\
p & =-\frac{1}{2} a_{i k} a_{k j} y_{i} y_{j}-c \sum_{i, k} a_{i k} y_{i}
\end{aligned}
$$

and with $\mathbf{S}$ given by eq(5).

For this work we shall consider $\mathbf{u}$ as being given by eq(6a), where $\mathbf{A}$ is a given matrix, and we shall prove the existence of a solution $\tilde{\psi}$, independent of $t$ and $\mathbf{y}$, to (1a)-(1b).

Before proceeding further, we pause for the following important observation. The solution $\tilde{\psi}$ to (1a)-(10) we inquire about - being a probability density - has to be non-trivial $(\tilde{\psi} \neq 0)$, non-negative and integrable. As $\tilde{\psi}=0$ is a solution to the aforementioned problem and as we have to mind about non-trivial ones, the solution non-uniqueness must be compulsory. Next, we know from [12, 13] that $\tilde{\psi}=0$ is the unique solution to (1a)-(1b) whenever $\mathbf{F}^{(c)}$ is an element of $L^{r}(B(0, \tilde{\delta})), r>n$. Therefore, what makes possible the existence of non-trivial solutions, is the fact that $\mathbf{F}^{(c)}$ is NOT an element of $L^{r}(B(0, \tilde{\delta})), r>n$ (in fact $\mathbf{F}^{(c)}$ is not an element of 
$L^{r}(B(0, \tilde{\delta}))$ for any $\left.r \geq 1\right)$

Now, as $\mathbf{F}^{(c)}=\nabla_{\tilde{\mathbf{x}}} U(\tilde{\mathbf{x}})$, with $U(\tilde{\mathbf{x}})=-\left(\tilde{\delta}^{2} / 2\right) \log \left(1-\|\tilde{\mathbf{x}}\|^{2} / \tilde{\delta}^{2}\right)$, equation (10) is usually re-written as (see [14]):

$$
-\frac{1}{2 \operatorname{De}} \nabla_{\tilde{\mathbf{x}}} \cdot\left[\tilde{M}(\tilde{\mathbf{x}}) \nabla_{\tilde{\mathbf{x}}}\left(\frac{\tilde{\psi}}{\tilde{M}}\right)\right]+\nabla_{\tilde{\mathbf{x}}} \cdot[\boldsymbol{\theta} \tilde{\mathbf{x}} \tilde{\psi}]=0
$$

where the function $\tilde{M}: B(0, \tilde{\delta}) \rightarrow \mathbb{R}$ is given by:

$$
\tilde{M}(\tilde{\mathbf{x}})=\frac{1}{J}\left(1-\frac{\|\tilde{\mathbf{x}}\|^{2}}{\tilde{\delta}^{2}}\right)^{\tilde{\delta}^{2} / 2}
$$

where $J$ is a normalization constant so that:

$$
\int_{B(0, \tilde{\delta})} \tilde{M}(\tilde{\mathbf{x}}) \mathrm{d} \tilde{\mathbf{x}}=1
$$

Next, for sake of generality, we replace $\boldsymbol{\theta} \tilde{\mathbf{x}}$ by an arbitrary function $\tilde{\mathbf{k}}: B(0, \tilde{\delta}) \rightarrow \mathbb{R}^{n}$, and the problem we focus on can be re-formulated as:

$$
\begin{aligned}
& -\frac{1}{2 \mathrm{De}} \nabla \cdot\left[\tilde{M} \nabla\left(\frac{\tilde{\psi}}{\tilde{M}}\right)\right]+\nabla \cdot(\tilde{\mathbf{k}} \tilde{\psi})=0, \quad \forall \tilde{\mathbf{x}} \in B(0, \tilde{\delta}) \\
& \left.\tilde{\psi}\right|_{\partial B(0, \tilde{\delta})}=0 \\
& \tilde{\psi} \geq 0 \\
& \int_{B(0, \tilde{\delta})} \tilde{\psi}(\tilde{\mathbf{x}}) \mathrm{d} \tilde{\mathbf{x}}=a
\end{aligned}
$$

where $a=\frac{1}{\operatorname{meas}(Q)}$ is a given constant.

Next, for notation convenience, we carry out the variable change $\mathbf{x}=\tilde{\mathbf{x}} / \tilde{\delta}$. This transforms the domain $\tilde{\Omega}$ into $\Omega=B(0,1)=\left\{\mathbf{x} \in \mathbb{R}^{n},\|\mathbf{x}\| \leq 1\right\}$. Let us denote $\psi(\mathbf{x})=\tilde{\psi}(\tilde{\mathbf{x}}), \mathbf{k}(\mathbf{x})=$ $2 \tilde{\delta} \operatorname{De} \tilde{\mathbf{k}}(\tilde{\mathbf{x}}), \delta=\tilde{\delta}^{2} / 2$ and let

$$
M: \Omega \rightarrow \mathbb{R}, M(\mathbf{x})=\left(1-\|\mathbf{x}\|^{2}\right)^{\delta} .
$$


Then, equations (10a)-(10d) become in $\Omega$ :

$$
\begin{aligned}
& -\nabla \cdot\left[M \nabla\left(\frac{\psi}{M}\right)\right]+\nabla \cdot(\mathbf{k} \psi)=0, \quad \forall \mathbf{x} \in \Omega \\
& \left.\psi\right|_{\partial \Omega}=0 \\
& \psi \geq 0 \\
& \int_{\Omega} \psi(\mathbf{x}) \mathrm{d} \mathbf{x}=b
\end{aligned}
$$

with $b>0$ and $\mathbf{k}: \Omega \rightarrow \mathbb{R}^{n}$ given. As in practical situations $\tilde{\delta}$ is (roughly speaking) close to 10 , then $\delta$ is close to 50 .

The goal of this paper is to prove the existence and uniqueness of a solution to the system of equations (11a)-(11d). We easily see that the aforementioned problem can be also formulated as following: prove that 0 is a simple eigenvalue of the operator (denoted from now on $L$ ) defined by the lhs of (11a) and the boundary condition (11b), with a corresponding non-negative and integrable eigenvector. In fact, we will prove that 0 is the principal eigenvalue of $L$ in the sense that the real part of any other eigenvalue of it is non-negative. To achieve this we use the classical Krein-Rutman theorems, in both weak and strong senses, on an appropriate operator obtained from $L$. This will also entail that $\psi$ is positive over $\Omega$ and behaves like $M$ on the boundary $\partial \Omega$.

The boundary value problem problem with unknowns $\mathbf{u}$ and $\tilde{\psi}$ as presented in (1a), (1b), (4a), (4b), (5) has attracted the attention of several investigators working in the area. For instance, in 15] Zhang and Zhang proved the existence of a local in time, regular solution to the system formed by equations (1a), (1b), (4a), (4b), and (5). The existence of a global in time solution was proved in [16] by Lin et al, and that in a particular case referred to as the "co-rotational" velocity field, that is, in equation (1a) the term $\boldsymbol{\theta}=(\nabla u)^{T}$ is replaced by $\boldsymbol{\theta}=\nabla_{y} u-\left(\nabla_{y} u\right)^{T}$. Moreover, for this same system of equations, in [17, Barret et al offered a proof for the existence and uniqueness of a solution to a regularized problem associated to the system (1a $),(1 \mathrm{~b}),(4 \mathrm{a}),(4 \mathrm{~b}),(5)$. Next, in 18 Lelièvre et al proved the existence and 
uniqueness of a local in time solution to the one dimensional motion system of equations in which the Fokker-Planck-Smoluchowski equation is replaced by a stochastic diffusion differential equation.

In [19] Du et al focused on the Fokker-Plank-Smoluchowski evolution equation only, assuming a steady and homogeneous macroscopic velocity field. For this they proved the global in time existence and uniqueness of a solution. For the corresponding steady state problem, the forementioned authors proved the existence of a solution only in the particular case where the tensor $\boldsymbol{\theta}$ in (1a) is either symmetric or antisymmetric. Some of their numerical simulations suggest the existence of steady-state solutions for arbitrary $\boldsymbol{\theta}$.

In this work we do prove the existence and uniqueness of steady state solutions for arbitrary $\theta$.

As an aside, in 14] Degond et al provided arguments in support of the validity of an asymptotic expansion solution, valid for small De numbers, first obtained in [1].

This paper is organized as follows:

- in Section 2 we state the main steady state existence and uniqueness result,

- Section 3 addresses some important functional analysis preliminaries,

- Section 1 is devoted to proving the conclusive existence and uniqueness result.

\section{Functional framework. Presentation of the main re-}

\section{sult.}

Let the following spaces be defined as:

$$
\begin{aligned}
& L_{M}^{2} \equiv L_{M}^{2}(\Omega):=\left\{u \in L_{\mathrm{loc}}^{1}(\Omega), \int_{\Omega} \frac{u^{2}}{M} \mathrm{~d} \mathbf{x}<\infty\right\} \\
& H_{M}^{1} \equiv H_{M}^{1}(\Omega):=\left\{u \in L_{\mathrm{loc}}^{1}(\Omega), \int_{\Omega}\left[\frac{u^{2}}{M}+M\left|\nabla\left(\frac{u}{M}\right)\right|^{2}\right] \mathrm{d} \mathbf{x}<\infty\right\}
\end{aligned}
$$


endowed with the norms

$$
\left(\int_{\Omega} \frac{u^{2}}{M} \mathrm{~d} \mathbf{x}\right)^{1 / 2} \quad \text { and respectively } \quad\left(\int_{\Omega}\left[\frac{u^{2}}{M}+M\left|\nabla\left(\frac{u}{M}\right)\right|^{2}\right] \mathrm{d} \mathbf{x}\right)^{1 / 2} .
$$

It is clear that $L_{M}^{2}$ is a Hilbert space. To see that $H_{M}^{1}$ is also a Hilbert space, let us remark that

$$
H_{M}^{1}=M \cdot \hat{H}_{M}^{1} \quad \text { with } \quad \hat{H}_{M}^{1}=\left\{v \in L_{\mathrm{loc}}^{1}(\Omega), \int_{\Omega}\left(M v^{2}+M|\nabla v|^{2}\right) \mathrm{d} \mathbf{x}<\infty\right\}
$$

It is well-known, as being a classical weighted Sobolev space, that $\hat{H}_{M}^{1}$ is complete (see for exemple Theorem 3.2.2.(a) in Triebel's monograph [20]) when endowed with the norm

$$
\left(\int_{\Omega}\left(M v^{2}+M|\nabla v|^{2}\right) \mathrm{d} \mathbf{x}\right)^{1 / 2}
$$

Since the application $\psi \in \hat{H}_{M}^{1} \rightarrow M \psi \in H_{M}^{1}$ is an isometry, we deduce that $H_{M}^{1}$ is complete.

For any $\varphi \in H_{M}^{1}(\Omega)$ we denote $|\varphi|_{1}$ the semi-norm on $H_{M}^{1}$ defined by $|\varphi|_{1}^{2}:=\int_{\Omega} M\left|\nabla \frac{\varphi}{M}\right|^{2} \mathrm{~d} \mathbf{x}$. Moreover, $\left(H_{M}^{1}(\Omega)\right)^{\prime}$ denotes the corresponding dual space and one has the canonical embedding

$$
L_{M}^{2} \subset\left(H_{M}^{1}(\Omega)\right)^{\prime}
$$

We now endeavor to search for solutions to equations (11a)-(11d) that are elements of $H_{M}^{1}$, as the trace on $\partial \Omega$ of any $u \in H_{M}^{1}(\Omega)$ is zero (see also Proposition 3.3 ).

To achieve this goal, equation ([1]) is first multiplied by $\varphi / M$, with $\varphi \in \mathcal{D}(\Omega)$ and next integrated over $\Omega$. It gives:

$$
\int_{\Omega} M \nabla\left(\frac{\psi}{M}\right) \cdot \nabla\left(\frac{\varphi}{M}\right) \mathrm{d} \mathbf{x}-\int_{\Omega} \mathbf{k} \psi \cdot \nabla\left(\frac{\varphi}{M}\right) \mathrm{d} \mathbf{x}=0
$$

Definition 2.1. $\psi \in H_{M}^{1}(\Omega)$ is a weak solution of the system (11a) $-(11 \mathrm{~d})$, provided that:

$$
\int_{\Omega} M \nabla\left(\frac{\psi}{M}\right) \cdot \nabla\left(\frac{\varphi}{M}\right) \mathrm{d} \mathbf{x}-\int_{\Omega} \mathbf{k} \psi \cdot \nabla\left(\frac{\varphi}{M}\right) \mathrm{d} \mathbf{x}=0, \quad \forall \varphi \in H_{M}^{1}(\Omega)
$$

and moreover, (11d) and (11d) are satisfied. 
Next, let the operator $L: H_{M}^{1}(\Omega) \rightarrow\left(H_{M}^{1}(\Omega)\right)^{\prime}$ be defined as:

$$
\langle L(u), \varphi\rangle:=\int_{\Omega} M \nabla\left(\frac{u}{M}\right) \cdot \nabla\left(\frac{\varphi}{M}\right) \mathrm{d} \mathbf{x}-\int_{\Omega} \mathbf{k} u \cdot \nabla\left(\frac{\varphi}{M}\right) \mathrm{d} \mathbf{x}, \quad \forall \varphi \in H_{M}^{1}(\Omega)
$$

Now, $L$ is well-defined, due to:

$$
\begin{aligned}
& \left|\int_{\Omega} M \nabla\left(\frac{u}{M}\right) \cdot \nabla\left(\frac{\varphi}{M}\right) \mathrm{d} \mathbf{x}-\int_{\Omega} \mathbf{k} u \cdot \nabla\left(\frac{\varphi}{M}\right) \mathrm{d} \mathbf{x}\right| \leq \\
& \leq \int_{\Omega}\left|M^{1 / 2} \nabla\left(\frac{u}{M}\right)\right| \cdot\left|M^{1 / 2} \nabla\left(\frac{\varphi}{M}\right)\right| \mathrm{d} \mathbf{x}+\int_{\Omega}|\mathbf{k}|\left|\frac{u}{M^{1 / 2}}\right| M^{1 / 2}\left|\nabla\left(\frac{\varphi}{M}\right)\right| \mathrm{d} \mathbf{x} \\
& \leq\|u\|_{H_{M}^{1}}\|\varphi\|_{H_{M}^{1}}+\|\mathbf{k}\|_{L^{\infty}}\|u\|_{L_{M}^{2}}\|\varphi\|_{H_{M}^{1}}
\end{aligned}
$$

It is now clear that our problem is tantamount to finding an element $\psi \in H_{M}^{1}$ such that

$$
\begin{aligned}
& L \psi=0 \\
& \psi \geq 0 \\
& \int_{\Omega} \psi(\mathbf{x}) \mathrm{d} \mathbf{x}=b,
\end{aligned}
$$

that is, $\psi$ must by a non-negative and integrable eigenvector of $L$ corresponding to the eigenvalue 0.

For any $\beta \geq 0$, let:

$$
X_{\beta}:=\left\{\varphi \in \mathcal{C}(\bar{\Omega}), \exists c \geq 0 \quad \text { s.t. }|\varphi(\mathbf{x})| \leq c M^{\beta}(\mathbf{x}), \forall \mathbf{x} \in \Omega\right\}
$$

$X_{\beta}$ is a Banach space endowed with the norm

$$
\|\varphi\|_{X_{\beta}}:=\sup _{\mathbf{x} \in \Omega} \frac{|\varphi(\mathbf{x})|}{M^{\beta}(\mathbf{x})}=\inf \left\{c \geq 0 \quad \text { s.t. } \quad|\varphi(\mathbf{x})| \leq c M^{\beta}(\mathbf{x}), \forall \mathbf{x} \in \Omega\right\} .
$$

Remark 2.1. $X_{0}=\mathcal{C}^{0}(\bar{\Omega})$, endowed with the usual norm $\|\varphi\|_{X_{0}}=\sup _{\mathbf{x} \in \Omega}|\varphi|$.

Remark 2.2. For $\beta_{1}<\beta_{2}$, the continuous inclusion $X_{\beta_{2}} \underset{\text { cont }}{\subset} X_{\beta_{1}}$ holds true.

Let the cone $P_{\beta} \subset X_{\beta}$ be defined as:

$$
P_{\beta}:=\left\{\varphi \in X_{\beta}, \varphi(\mathbf{x}) \geq 0, \forall \mathbf{x} \in \Omega\right\}
$$


It is clear that $P_{\beta}$ is a reproducible cone for the space $X_{\beta}$, that is $X_{\beta}=P_{\beta}-P_{\beta}$.

It can be easily seen the interior $\stackrel{\circ}{P}_{\beta}$ of $P_{\beta}$ is given by:

$$
\begin{aligned}
\stackrel{\circ}{P}_{\beta} & =\left\{\varphi \in X_{\beta} \quad \text { s.t. } \quad \inf _{\mathbf{x} \in \Omega} \frac{\varphi(\mathbf{x})}{M^{\beta}(\mathbf{x})}>0\right\}=\left\{\varphi \in X_{\beta}, \exists c_{1}>0 \quad \text { s.t. } \quad \varphi(\mathbf{x}) \geq c_{1} M^{\beta}(\mathbf{x}), \forall \mathbf{x} \in \Omega\right\} \\
& =\left\{\varphi \in \mathscr{C}(\Omega), \exists c_{1}, c_{2} ; 0<c_{1}<c_{2}, \quad \text { s.t. } \quad c_{1} M^{\beta}(\mathbf{x}) \leq \varphi(\mathbf{x}) \leq c_{2} M^{\beta}(\mathbf{x}), \forall \mathbf{x} \in \Omega\right\} . \quad(21)
\end{aligned}
$$

We now state the cornerstone result of this paper:

Theorem 2.1. [Existence and uniqueness theorem] Let $b>0, \delta \geq 8$ and $\mathbf{k} \in\left(W^{1, \infty}(\Omega)\right)^{n}$. Then there exists an unique solution $\psi$ to the system ((17a)-(17d)). Moreover, this solution belongs to $\stackrel{\circ}{P}_{1}$ which amounts to say that $\psi$ is continuous in $\Omega$, and there exist $c_{1}, c_{2}$ with $0<c_{1}<c_{2}$ such that

$$
c_{1} M(\mathbf{x}) \leq \psi(\mathbf{x}) \leq c_{2} M(\mathbf{x}), \quad \forall \mathbf{x} \in \Omega
$$

Remark 2.3. We assume throughout this paper that $\delta$ and $\mathbf{k}$ comply with the hypotheses of Theorem 2.1. Given the physical model under consideration (for which we gave a suitable description in the Introduction section), such an assumption does not lower down the level of generality.

\section{Several preliminary results}

\subsection{Basic facts}

In the following we denote for any real $\alpha$ the operator $L_{\alpha}: H_{M}^{1}(\Omega) \rightarrow\left(H_{M}^{1}(\Omega)\right)^{\prime}$ given by $L_{\alpha}=L+\alpha I_{d}, I_{d}$ being the identity operator.

It is assumed, throughout this paper, that $\alpha$ is large enough so that:

$$
\alpha \geq \max \left\{\frac{1}{2}\|\mathbf{k}\|_{L^{\infty}(\Omega)}+1, \quad 4 \lambda_{0}^{2}+\lambda_{0} n+2 \lambda_{0}\|\mathbf{k}\|_{L^{\infty}(\Omega)}+\|\nabla \cdot \mathbf{k}\|_{L^{\infty}(\Omega)}\right\}
$$

where

$$
\lambda_{0}=2\left(\|\mathbf{k}\|_{L^{\infty}(\Omega)}+1\right)
$$


Proposition 3.1. The operator $L_{\alpha}$ is invertible.

Proof. Let $f \in\left(H_{M}^{1}(\Omega)\right)^{\prime}$, arbitrary. We have to prove the existence of a unique solution $u \in H_{M}^{1}$ to

$$
a_{\alpha}(u, \varphi)=\langle f, \varphi\rangle, \forall \varphi \in H_{M}^{1}
$$

where, in the above,

$$
a_{\alpha}(u, \varphi)=\int_{\Omega} M \nabla\left(\frac{u}{M}\right) \cdot \nabla\left(\frac{\varphi}{M}\right) \mathrm{d} \mathbf{x}-\int_{\Omega} \mathbf{k} u \cdot \nabla\left(\frac{\varphi}{M}\right) \mathrm{d} \mathbf{x}+\alpha \int_{\Omega} \frac{u \varphi}{M} \mathrm{~d} \mathbf{x}
$$

Next, to use the Lax-Milgram theorem, one only needs to prove $a_{\alpha}$ is coercive as all other theorem constitutive assumptions are obviously fulfilled.

The fact that $a_{\alpha}$ is coercive is an immediat consequence of the inequality

$$
\begin{aligned}
& \left|\int_{\Omega} \mathbf{k} \varphi \cdot \nabla\left(\frac{\varphi}{M}\right) \mathrm{d} \mathbf{x}\right| \leq \int_{\Omega}|\mathbf{k}|\left|\frac{\varphi}{M^{1 / 2}}\right|\left|M^{1 / 2} \nabla\left(\frac{\varphi}{M}\right)\right| \mathrm{d} \mathbf{x} \\
& \leq \frac{1}{2}\left(|\varphi|_{1}^{2}+\|\mathbf{k}\|_{L^{\infty}}^{2}\|\varphi\|_{L_{M}^{2}}^{2}\right)
\end{aligned}
$$

and of the choice of $\alpha$.

Let then $B_{\alpha}:\left(H_{M}^{1}\right)^{\prime} \rightarrow H_{M}^{1}$ denote the inverse operator of $L_{\alpha}$.

Clearly

$$
B_{\alpha} \in \mathcal{L}\left(\left(H_{M}^{1}\right)^{\prime}, H_{M}^{1}\right)
$$

and, also,

$$
B_{\alpha} \in \mathcal{L}\left(L_{M}^{2}, L_{M}^{2}\right)
$$

Lemma 3.1. [Weak Maximum Principle] Let $f \in\left(H_{M}^{1}\right)^{\prime}, f \geq 0$, and $u=B_{\alpha} f$. Then $u \geq 0$.

Proof. The proof is classical and consists to choose $\varphi=u^{-}$in the corresponding variational formulation. (see for exemple [21] for the non-degenerate case $M \equiv 1$ ). 
Lemma 3.2. [Comparison Principle] Let $\Omega^{\prime}$ be an open set such that $\Omega^{\prime} \subset \Omega$, and let $\overline{\Omega^{\prime}}$ denote its closure. Let $u, v \in H_{M}^{1}(\Omega)$ so that $L_{\alpha} u$ and $L_{\alpha} v$ be functions well defined on $\Omega^{\prime}$. Assume that $L_{\alpha} u \geq L_{\alpha} v$ on $\Omega^{\prime}$. Then:

- Case 1. If $\overline{\Omega^{\prime}} \subset \Omega$ and if $u \geq v$ on $\partial \Omega^{\prime}$, then $u \geq v$ on $\Omega^{\prime}$.

- Case 2. If $\overline{\Omega-\Omega^{\prime}} \subset \Omega$ and if $u \geq v$ on $\partial\left(\Omega-\Omega^{\prime}\right)$, then $u \geq v$ on $\Omega^{\prime}$.

Proof. Let $w=u-v \in H_{M}^{1}(\Omega)$ and $f=L_{\alpha}(u-v)$. Then, for $\forall \varphi \in H_{M}^{1}(\Omega)$, such that $\left.\varphi\right|_{\Omega-\Omega^{\prime}}=0$, one has:

$$
\int_{\Omega^{\prime}} M \nabla\left(\frac{w}{M}\right) \cdot \nabla\left(\frac{\varphi}{M}\right) \mathrm{d} \mathbf{x}^{\prime}-\int_{\Omega^{\prime}} \mathbf{k} w \cdot \nabla\left(\frac{\varphi}{M}\right) \mathrm{d} \mathbf{x}^{\prime}+\alpha \int_{\Omega^{\prime}} \frac{w \varphi}{M} \mathrm{~d} \mathbf{x}^{\prime}=\int_{\Omega^{\prime}} \frac{f \varphi}{M} \mathrm{~d} \mathbf{x}^{\prime}
$$

We now take in $27 \varphi: \Omega \rightarrow \mathbb{R}$ defined by

$$
\varphi=\left\{\begin{array}{ccc}
w^{-} & \text {on } & \Omega^{\prime} \\
0 & \text { on } & \Omega-\Omega^{\prime}
\end{array}\right.
$$

and we easily obtain the result.

We now introduce for any $\beta>0$

$$
L_{2, \beta}:=\left\{\varphi \in L_{\mathrm{loc}}^{1}(\Omega) \quad \text { s.t. } \quad \frac{\varphi}{M^{\beta}} \in L_{2}(\Omega)\right\} .
$$

Actually, $L_{2, \beta}(\Omega)$ is a Hilbert space endowed with the norm

$$
\|\varphi\|_{L_{2, \beta}(\Omega)}=\left\|\frac{\varphi}{M^{\beta}}\right\|_{L_{2}(\Omega)}, \forall \varphi \in L_{2, \beta}(\Omega)
$$

We have, as a straightforward consequence of Theorem 6.2.5 of [24], the following continuous inclusion:

$$
H_{M}^{1}(\Omega) \underset{\text { cont }}{\subset} L_{2,1 / 2+1 / \delta}(\Omega)
$$

Next: 
Proposition 3.2. (a) If $\beta>1 / 2-1 /(2 \delta)$, then $X_{\beta} \underset{\text { cont }}{\subset} L_{M}^{2}$.

(b) If $\beta>1 / 2-3 /(2 \delta)$, then $X_{\beta} \underset{\text { cont }}{\subset}\left(H_{M}^{1}\right)^{\prime}$.

Proof. (a) Let $\varphi \in X_{\beta}$, arbitrarily. Then, $|\varphi(\mathbf{x})| \leq M^{\beta}(\mathbf{x})\|\varphi\|_{X_{\beta}}$, from which we get that:

$$
\int_{\Omega} \frac{\varphi^{2}}{M} \mathrm{~d} \mathbf{x} \leq\|\varphi\|_{X_{\beta}}^{2} \int_{\Omega} \frac{M^{2 \beta}}{M} \mathrm{~d} \mathbf{x}=\|\varphi\|_{X_{\beta}}^{2} \int_{\Omega}\left(1-\|\mathbf{x}\|^{2}\right)^{(2 \beta-1) \delta} \mathrm{d} \mathbf{x}
$$

However, $\int_{\Omega}\left(1-\|\mathbf{x}\|^{2}\right)^{(2 \beta-1) \delta} \mathrm{d} \mathbf{x}<+\infty$ iff $\beta>1 / 2-1 /(2 \delta)$.

(b) Let $\varphi \in X_{\beta}$ and $\psi \in H_{M}^{1}$ arbitrarily. We have

$$
\left|\int_{\Omega} \frac{\varphi \psi}{M} \mathrm{~d} \mathbf{x}\right| \leq\|\varphi\|_{X_{\beta}} \int_{\Omega} M^{\beta-1}|\psi| \mathrm{d} \mathbf{x}
$$

However:

$$
\int_{\Omega} M^{\beta-1}|\psi| \mathrm{d} \mathbf{x} \leq \underbrace{\left\|M^{-1 / 2-1 / \delta} \psi\right\|_{L^{2}(\Omega)}}_{=\|\psi\|_{L_{2,1 / 2+1 / \delta}}}\left\|M^{\beta-1 / 2+1 / \delta}\right\|_{L^{2}(\Omega)}
$$

Moreover, the $L^{2}$-norm of $M^{\beta-1 / 2+1 / \delta}$ is finite iff $\beta>1 / 2-3 /(2 \delta)$.

Using also the continuous inclusion (31) we have the result stated.

Proposition 3.3. Let $\beta$ be such that $0 \leq \beta \leq 1 / 2-1 / \delta$. Then, for any $u \in H_{M}^{1}, \frac{u}{M^{\beta}} \in H_{0}^{1}(\Omega)$; moreover, $\left\|\frac{u}{M^{\beta}}\right\|_{H^{1}} \leq c\|u\|_{H_{M}^{1}}$.

Proof. Let $v=\frac{u}{M^{\beta}}=\frac{u}{M^{1 / 2}} M^{1 / 2-\beta}$. We actually need to prove that $\frac{u}{M^{1 / 2}} \in H^{1}(\Omega)$. From this, since $M^{1 / 2-\beta} \in \mathscr{C}^{1}(\bar{\Omega})$ and $\left.M^{1 / 2-\beta}\right|_{\partial \Omega}=0$, it will follow that $v \in H_{0}^{1}(\Omega)$.

To begin with, notice first that $\frac{u}{M^{1 / 2}} \in L^{2}(\Omega)$, as $u \in L_{M}^{2}(\Omega)$. Next,

$$
\nabla\left(\frac{u}{M^{1 / 2}}\right)=\nabla\left(M^{1 / 2} \frac{u}{M}\right)=M^{1 / 2} \nabla\left(\frac{u}{M}\right)+\nabla\left(M^{1 / 2}\right) \frac{u}{M} .
$$

Now, $M^{1 / 2} \nabla\left(\frac{u}{M}\right) \in L^{2}(\Omega)$ as $u \in H_{M}^{1}$. Let us next show that $\nabla\left(M^{1 / 2}\right) \frac{u}{M} \in L^{2}(\Omega)$. One has: 


$$
\nabla\left(M^{1 / 2}\right) \frac{u}{M}=\frac{\nabla M}{2 M^{3 / 2}} u=\frac{u}{M^{1 / 2+1 / \delta}} \underbrace{\frac{\nabla M}{2 M^{1-1 / \delta}}}_{\in L^{\infty}(\Omega)} .
$$

Next, by (31) $\frac{u}{M^{1 / 2+1 / \delta}} \in L^{2}(\Omega)$, from which we infer that $\frac{u}{M^{1 / 2}} \in H^{1}(\Omega)$, and further on that $\frac{u}{M^{\beta}} \in H_{0}^{1}(\Omega)$. It is easily deduced that:

$$
\left\|\frac{u}{M^{\beta}}\right\|_{H_{0}^{1}} \leq c\|u\|_{H_{M}^{1}}
$$

Remark 3.1. Taking $\beta=0$ in Proposition 3.3 we deduce that $u \in H_{0}^{1}(\Omega)$ whenever $u \in H_{M}^{1}(\Omega)$, which triggers that the trace of $u$ on the boundary $\partial \Omega$ is equal to zero.

Proposition 3.4. Let $\varphi \in X_{\beta}, \beta>1 / 2+1 /(2 \delta)$, be such that $\nabla \varphi \in\left(X_{\gamma}\right)^{n}$ with $\gamma>1 / 2-1 /(2 \delta)$. Then $\varphi \in H_{M}^{1}$.

Proof. From Proposition 3.2 a) we have $\varphi \in L_{M}^{2}$. Next,

$$
\begin{aligned}
\int_{\Omega} M\left|\nabla\left(\frac{\varphi}{M}\right)\right|^{2} \mathrm{~d} \mathbf{x} & =\int_{\Omega} M\left|\frac{1}{M} \nabla \varphi-\frac{\nabla M}{M^{2}} \varphi\right|^{2} \mathrm{~d} \mathbf{x} \\
& \leq 2 \int_{\Omega} \frac{1}{M}|\nabla \varphi|^{2} \mathrm{~d} \mathbf{x}+2 \int_{\Omega} \frac{1}{M^{3}}|\nabla M|^{2} \varphi^{2} \mathrm{~d} \mathbf{x}
\end{aligned}
$$

However,

$$
\int_{\Omega} \frac{1}{M}|\nabla \varphi|^{2} \mathrm{~d} \mathbf{x} \leq c \int_{\Omega} \frac{1}{M} M^{2 \gamma} \mathrm{d} \mathbf{x}<+\infty
$$

provided that $\delta(2 \gamma-1)>-1$, which amounts to $\gamma>1 / 2-1 /(2 \delta)$.

Next,

$$
\begin{aligned}
\int_{\Omega} \frac{|\nabla M|^{2} \varphi^{2}}{M^{3}} \mathrm{~d} \mathbf{x} \leq c_{1} \int_{\Omega} \frac{|\nabla M|^{2} M^{2 \beta}}{M^{3}} \mathrm{~d} \mathbf{x} & \leq c_{2} \int_{\Omega} \frac{\left(1-\|\mathbf{x}\|^{2}\right)^{2(\delta-1)}}{\left(1-\|\mathbf{x}\|^{2}\right)^{3 \delta}}\left(1-\|\mathbf{x}\|^{2}\right)^{2 \delta \beta} \mathrm{d} \mathbf{x} \\
& \leq c_{2} \int_{\Omega}\left(1-\|\mathbf{x}\|^{2}\right)^{2 \delta \beta+2 \delta-2-3 \delta} \mathrm{d} \mathbf{x}
\end{aligned}
$$


For the above equation (40) to hold true it is necessary that $2 \delta \beta-\delta-2>-1$, i.e. $\beta>$ $1 / 2+1 /(2 \delta)$

Since $M^{\beta} \in X_{\beta}$ for any $\beta \geq 0$ we have the following direct consequence of the above result:

Proposition 3.5. For any $\beta>1 / 2+1 /(2 \delta)$, we have $M^{\beta} \in H_{M}^{1}$.

\subsection{Continuity and compactness properties of $B_{\alpha}$}

The goal now is to appropriately introduce several $\left(Y^{\prime}, Y^{\prime \prime}\right)$ paires of Banach spaces such that $B_{\alpha}$ is well defined and continuous from $Y^{\prime}$ to $Y^{\prime \prime}$. Some compactness properties of $B_{\alpha}$, needed further on, are also proved.

Lemma 3.3. (i) Let $\beta_{2} \in \mathbb{R}$ such that $1 / 2-3 /(2 \delta)<\beta_{2} \leq 1 / 2-1 / \delta$. Then $B_{\alpha} \in \mathcal{L}\left(L_{M}^{2}, X_{\beta_{2}}\right)$. Moreover, $B_{\alpha}$ is a compact application from $L_{M}^{2}$ onto $X_{\beta_{2}}$.

(ii) Let $\beta_{1}, \beta_{2} \in \mathbb{R}$ such that $1 / 2-3 /(2 \delta)<\beta_{2} \leq 1 / 2-1 / \delta$, and $\beta_{1} \geq \beta_{2}$. Then $B_{\alpha} \in$ $\mathcal{L}\left(X_{\beta_{1}}, X_{\beta_{2}}\right)$. Moreover, $B_{\alpha}$ is a compact application from $X_{\beta_{1}}$ onto $X_{\beta_{2}}$.

Proof. The proofs for the above two statements are pretty much similar in nature; henceforth, we offer below a global proof, and pause wherever necessary to particularize it so to get the results in either (i) or (ii). Keeping that in mind, let $f \in L_{M}^{2}$ (for the (i) part) and $f \in X_{\beta_{1}}$ (for the (ii) part) and let $u=B_{\alpha} f$. Observe that:

$$
L_{M}^{2} \underset{\text { cont. }}{\subset}\left(H_{M}^{1}\right)^{\prime}
$$

and that

$$
X_{\beta_{1}} \underset{\text { cont. }}{\subset}\left(H_{M}^{1}\right)^{\prime}
$$


as consequences of Proposition 3.2. In both cases $f \in\left(H_{M}^{1}\right)^{\prime}$ and $u \in H_{M}^{1}$ solves the equation

$$
-\nabla \cdot\left[M \nabla\left(\frac{u}{M}\right)\right]+\nabla \cdot(\mathbf{k} u)+\alpha u=f, u \in H_{M}^{1}
$$

We also have

$$
\|u\|_{H_{M}^{1}} \leq c\|f\|_{L_{M}^{2}} \quad \text { for the part (i) }
$$

and

$$
\|u\|_{H_{M}^{1}} \leq c\|f\|_{X_{\beta_{1}}} \quad \text { for the part (ii). }
$$

Denote $v(\mathbf{x})=u(\mathbf{x}) / M^{\beta_{2}}(\mathbf{x})$; we first take on to prove that $v$ is bounded on $\Omega$, which prompts that $u$ belongs to $X_{\beta_{2}}$.

Making use of the fact that $u=v M^{\beta_{2}}$ into (43) leads to:

$$
\begin{aligned}
& -\nabla \cdot\left[M^{\beta_{2}}(\nabla v)+\left(\beta_{2}-1\right) M^{\beta_{2}-1}(\nabla M) v\right]+(\nabla \cdot \mathbf{k}) M^{\beta_{2}} v \\
& +\beta_{2} M^{\beta_{2}-1}(\nabla M) \cdot \mathbf{k} v+\mathbf{k} M^{\beta_{2}} \cdot(\nabla v)+\alpha M^{\beta_{2}} v=f, \forall \mathbf{x} \in \Omega
\end{aligned}
$$

which, after a few re-arrangements, can be re-written as:

$$
-\triangle v=g
$$

where

$$
\begin{aligned}
g & =\frac{f}{M^{\beta_{2}}}+\left[\left(2 \beta_{2}-1\right) \frac{\nabla M}{M}-\mathbf{k}\right] \cdot \nabla v \\
& +\left[\left(\beta_{2}-1\right) \frac{\triangle M}{M}+\left(\beta_{2}-1\right)^{2} \frac{|\nabla M|^{2}}{M^{2}}-\nabla \cdot \mathbf{k}-\beta_{2} \frac{\nabla M}{M} \cdot \mathbf{k}-\alpha\right] v
\end{aligned}
$$

We also deduce from Proposition 3.3 that

$$
v \in H_{0}^{1}(\Omega)
$$


In the following we shall obtain some convenient estimates for the function $g$. We have

$$
\begin{aligned}
\nabla v & =\nabla\left(\frac{u}{M} M^{1-\beta_{2}}\right)=M^{1-\beta_{2}} \nabla\left(\frac{u}{M}\right)+\nabla\left(M^{1-\beta_{2}}\right) \frac{u}{M} \\
& =M^{1 / 2-\beta_{2}} \underbrace{M^{1 / 2} \nabla\left(\frac{u}{M}\right)}_{\in L^{2}(\Omega) \text { as } u \in H_{M}^{1}}+\left(1-\beta_{2}\right) M^{-\beta_{2}-1}(\nabla M) u .
\end{aligned}
$$

Using equation (46) we get:

$$
g=\frac{f}{M^{\beta_{2}}}+M^{1 / 2} g_{1}(\mathbf{x}) \cdot \nabla\left(\frac{u}{M}\right)+g_{2}(\mathbf{x}) u
$$

where, in the above,

$$
\begin{aligned}
g_{1}(\mathbf{x}) & =\left[\left(2 \beta_{2}-1\right) \frac{\nabla M}{M}-\mathbf{k}\right] M^{1 / 2-\beta_{2}} \\
g_{2}(\mathbf{x}) & =\left(1-\beta_{2}\right)\left[\left(2 \beta_{2}-1\right) \frac{\nabla M}{M}-\mathbf{k}\right] \cdot \frac{\nabla M}{M^{\beta_{2}+1}} \\
& +\frac{1}{M^{\beta_{2}}}\left[\left(\beta_{2}-1\right) \frac{\triangle M}{M}+\left(\beta_{2}-1\right) \frac{|\nabla M|^{2}}{M^{2}}-\nabla \cdot \mathbf{k}-\beta_{2} \frac{\nabla M}{M} \cdot \mathbf{k}-\alpha\right]
\end{aligned}
$$

For the (i) part of Lemma 3.3 one has:

$$
\left\|\frac{f}{M^{\beta_{2}}}\right\|_{L^{2}} \leq\left\|M^{1 / 2-\beta_{2}}\right\|_{L^{\infty}}\|f\|_{L_{M}^{2}}
$$

while for the (ii) part of Lemma 3.3 one gets:

$$
\left\|\frac{f}{M^{\beta_{2}}}\right\|_{L^{2}} \leq\left\|M^{\beta_{1}-\beta_{2}}\right\|_{L^{\infty}}\|f\|_{X_{\beta_{1}}}
$$

Moreover,

$$
\frac{\nabla M}{M} \underset{\|\mathbf{x}\| \rightarrow 1}{\sim} \frac{1}{1-\|\mathbf{x}\|^{2}}=\frac{1}{M^{1 / \delta}}
$$

Therefore, the above leads to $g_{1} \in L^{\infty}(\Omega)$. We then deduce $g_{1} M^{1 / 2} \nabla(u / M) \in L^{2}(\Omega)$ and

$$
\left\|g_{1} M^{1 / 2} \nabla(u / M)\right\|_{L^{2}(\Omega)} \leq c_{1}\|u\|_{H_{M}^{1}}
$$

Now, observe that:

$$
g_{2}(\mathbf{x}) \underset{\|\mathbf{x}\| \rightarrow 1}{\sim} \frac{1}{M^{\beta_{2}+2 / \delta}}
$$


which implies

$$
g_{2}(\mathbf{x}) u \underset{\|\mathbf{x}\| \rightarrow 1}{\sim} \frac{u}{M^{1 / 2+1 / \delta}} \underbrace{M^{1 / 2-\beta_{2}-1 / \delta}}_{\in L^{\infty}, \text { as } \beta_{2} \leq 1 / 2-1 \delta} .
$$

We deduce with the help of inclusion (31) that

$$
\left\|g_{2} u\right\|_{L^{2}(\Omega)} \leq c_{1}\|u\|_{H_{M}^{1}}
$$

and further on, from (49), (50), (51), (52) and (53), that

$$
\|g\|_{L^{2}(\Omega)} \leq c\|f\|_{L_{M}^{2}}
$$

for part (i), and

$$
\|g\|_{L^{2}(\Omega)} \leq c\|f\|_{X_{\beta_{1}}}
$$

for (ii) part.

Now since $v$ satisfies (46) and (47) we obtain $v \in H^{2}(\Omega)$, and $\|v\|_{H^{2}(\Omega)} \leq c\|g\|_{L^{2}(\Omega)}$. By the Sobolev's inclusion $H^{2}(\Omega) \underset{\text { cont }}{\subset} \mathcal{C}(\bar{\Omega}), n=2,3$ it follows that $v \in \mathcal{C}(\bar{\Omega})$, and that $\|v\|_{\mathcal{C}(\bar{\Omega})} \leq$ $c_{1}\|g\|_{L^{2}(\Omega)}$.

Thus $u \in X_{\beta_{2}}$ and

$$
\|u\|_{X_{\beta_{2}}} \leq c_{1}\|g\|_{L^{2}(\Omega)}
$$

Next, making use of (54) and (55), we deduce, for part (i) that $B_{\alpha} \in \mathscr{L}\left(L_{M}^{2}, X_{\beta_{2}}\right)$, and for part (ii) that $B_{\alpha} \in \mathscr{L}\left(X_{\beta_{1}}, X_{\beta_{2}}\right)$.

In order to show the compactness of $B_{\alpha}$, let $\left(f_{q}\right)_{q \in \mathbb{N}}$ be a bounded sequence in $L_{M}^{2}$ for part (i), and in $X_{\beta_{1}}$ for part (ii), respectively. Denote $u_{q}=B_{\alpha}\left(f_{q}\right) \in X_{\beta_{2}}$, and $v_{q}=u_{q} / M^{\beta_{2}}$. Next it is proved that $v_{q}$ is bounded in $H^{2}(\Omega)$. As the domain $\Omega$ is bounded, the inclusion $H^{2}(\Omega) \subset \mathcal{C}(\bar{\Omega})$ is compact; hence there exists a subsequence $q^{\prime}$ of $q$ and a $v \in \mathcal{C}(\bar{\Omega})$ such that $v_{q^{\prime}}^{\underset{\mathcal{C}(\bar{\Omega})}{\rightarrow}} v$. Denoting $u=v M^{\beta_{2}}$, we have that $u \in X_{\beta_{2}}$ and $\sup _{\mathbf{x} \in \Omega} \frac{\left|v_{q^{\prime}}(\mathbf{x})-v(\mathbf{x})\right|}{M^{\beta_{2}}(\mathbf{x})} \underset{q^{\prime} \rightarrow+\infty}{\rightarrow} 0$. Therefore $\underset{\substack{q^{\prime} \rightarrow+\infty \\ q^{\prime} \rightarrow+\infty}}{u}$ with respect to the $X_{\beta_{2}}$ space topology. 
For any $r>0$, let us denote $\Omega_{r}:=\{\mathbf{x}:\|\mathbf{x}\|<r\} \equiv B(0, r)$.

Lemma 3.4. Let $\beta$ be such that $1 / 2+1 /(2 \delta)<\beta<1$. Then $B_{\alpha} \in \mathscr{L}\left(X_{\beta-2 / \delta}, X_{\beta}\right)$.

Proof. Proposition 3.2 b) gives that $X_{\beta-2 / \delta} \in\left(H_{M}^{1}\right)^{\prime}($ as $1 / 2-3 /(2 \delta)<\beta-2 / \delta)$, which entails that the operator $B_{\alpha}$ is well defined over $X_{\beta-2 / \delta}$. Let $f \in X_{\beta-2 / \delta}$ and $u=B_{\alpha}(f)$. We have to prove the validity of the following assertion:

There exists $A^{\prime}>0$ independent on $f$ such that

$$
A M^{\beta}(\mathbf{x})-u(\mathbf{x}) \geq 0, \quad \forall \mathbf{x} \in \Omega
$$

and

$$
A M^{\beta}(\mathbf{x})+u(\mathbf{x}) \geq 0, \quad \forall \mathbf{x} \in \Omega
$$

where we denoted $A=A^{\prime}\|f\|_{\beta-2 / \delta}$. We shall provide a proof for the first one only, i.e. for (57), as the other may be proved similarly. The proof for (57) relies on the Comparison Principle stated in Lemma 3.2 .

One has:

$$
\begin{aligned}
L_{\alpha}\left(M^{\beta}\right) & =M^{\beta}(\alpha+\nabla \cdot \mathbf{k})+M^{\beta-1}[\beta \mathbf{k} \cdot \nabla M+(1-\beta) \Delta M] \\
& -M^{\beta-2}(1-\beta)^{2}|\nabla M|^{2}
\end{aligned}
$$

As:

$$
\begin{aligned}
& \nabla M=-2 \delta \mathbf{x}\left(1-\|\mathbf{x}\|^{2}\right)^{\delta-1} \\
& \Delta M=-2 n \delta\left(1-\|\mathbf{x}\|^{2}\right)^{\delta-1}+4 \delta(\delta-1)\|\mathbf{x}\|^{2}\left(1-\|\mathbf{x}\|^{2}\right)^{\delta-2}
\end{aligned}
$$

then,

$$
\begin{aligned}
L_{\alpha}\left(M^{\beta}\right) & =a_{0}(\mathbf{x})\left(1-\|\mathbf{x}\|^{2}\right)^{\delta \beta-2}+a_{1}(\mathbf{x})\left(1-\|\mathbf{x}\|^{2}\right)^{\delta \beta-1}+a_{2}(\mathbf{x})\left(1-\|\mathbf{x}\|^{2}\right)^{\delta \beta}, \text { where } \\
a_{0}(\mathbf{x}) & =4 \delta(1-\beta)(\delta \beta-1)\|\mathbf{x}\|^{2} \\
a_{1}(\mathbf{x}) & =-[2 \delta \beta \mathbf{x} \cdot \mathbf{k}+2(1-\beta) n \delta] \\
a_{2}(\mathbf{x}) & =\alpha+\nabla \cdot k(\mathbf{x})
\end{aligned}
$$


It is clear that $a_{0}(\mathbf{x}) \geq 0, \forall \mathbf{x} \in \Omega$. Next, since $f \in X_{\beta-2 / \delta}$, we deduce

$$
-f(\mathbf{x}) \geq-\|f\|_{X_{\beta-2 / \delta}} M^{\beta-2 / \delta}(\mathbf{x})=-\|f\|_{X_{\beta-2 / \delta}}\left(1-\|\mathbf{x}\|^{2}\right)^{\delta \beta-2}
$$

Then:

$$
\begin{aligned}
A L_{\alpha}\left(M^{\beta}\right)-f & \geq\left[A a_{0}(\mathbf{x})-\|f\|_{X_{\beta-2 / \delta}}\right]\left(1-\|\mathbf{x}\|^{2}\right)^{\delta \beta-2}+A a_{1}(\mathbf{x})\left(1-\|\mathbf{x}\|^{2}\right)^{\delta \beta-1} \\
& +A a_{2}(\mathbf{x})\left(1-\|\mathbf{x}\|^{2}\right)^{\delta \beta}
\end{aligned}
$$

In the following, we restrict ourselves to $\Omega-\Omega_{1 / 2}$, henceforth $\|\mathbf{x}\| \geq 1 / 2$. Then

$$
a_{0}(\mathbf{x}) \geq a_{0}^{0}:=\delta(1-\beta)(\delta \beta-1)>0, \quad \forall \mathbf{x} \in \Omega-\Omega_{1 / 2}
$$

and

$$
\begin{aligned}
A L_{\alpha}\left(M^{\beta}\right)-f & \geq A a_{0}^{0}\left(1-\|\mathbf{x}\|^{2}\right)^{\delta \beta-2}\left[1-\frac{\|f\|_{X_{\beta-2 / \delta}}}{A a_{0}^{0}}+\frac{a_{1}(\mathbf{x})}{a_{0}^{0}}\left(1-\|\mathbf{x}\|^{2}\right)\right. \\
& \left.+\frac{a_{2}(\mathbf{x})}{a_{0}^{0}}\left(1-\|\mathbf{x}\|^{2}\right)^{2}\right], \quad \forall \mathbf{x} \in \Omega-\Omega_{1 / 2}
\end{aligned}
$$

Assume $f \neq 0$ (this is not too restrictive as, whenever $f=0$, the inequality (57) is satisfied with $A=0$ ).

Let us choose $r_{0} \in\left[\frac{1}{2}, 1[\right.$ close enough to 1 such that

$$
\left\{\begin{array}{l}
\frac{1-r_{0}^{2}}{a_{0}^{0}} \sup _{\mathbf{x} \in \Omega}\left|a_{1}(\mathbf{x})\right| \leq \frac{1}{4} \\
\frac{\left(1-r_{0}^{2}\right)^{2}}{a_{0}^{0}} \sup _{\mathbf{x} \in \Omega}\left|a_{2}(\mathbf{x})\right| \leq \frac{1}{4}
\end{array}\right.
$$

On the other hand, from Lemma 3.3 (ii) with $\beta_{1}=\beta-2 / \delta$ and $\beta_{2}=\min \{\beta-2 / \delta, 1 / 2-1 / \delta\}$ we deduce $u \in X_{\beta_{2}}$ (since $\left.f \in X_{\beta_{1}}\right)$ and

$$
\|u\|_{\mathcal{C}(\bar{\Omega})} \leq c_{1}\|u\|_{X_{\beta_{2}}} \leq c_{2}\|f\|_{X_{\beta-2 / \delta}}
$$

Take now

$$
A=\max \left\{\frac{4}{a_{0}^{0}}, \frac{c_{2}}{\left(1-r_{0}^{2}\right)^{\delta \beta}}\right\}\|f\|_{X_{\beta-2 / \delta}} .
$$

Clearly, from (60), (61) and (62),

$$
L_{\alpha}\left(A M^{\beta}-u\right)=A L_{\alpha}\left(M^{\beta}\right)-f \geq 0 \quad \text { on } \Omega-\Omega_{r_{0}}
$$


and

$$
A M^{\beta} \geq u \quad \text { on } \bar{\Omega}_{r_{0}} .
$$

respectively. Invoking the Comparison Principle (Lemma 3.2) and the fact that $u, M^{\beta} \in H_{M}^{1}$, leads to

$$
A M^{\beta} \geq u \quad \text { on } \Omega-\Omega_{r_{0}}, \quad \text { which implies } \quad A M^{\beta} \geq u \text { on } \Omega \text {. }
$$

This ends the proof.

Lemma 3.5. $B_{\alpha} \in \mathscr{L}\left(X_{1-1 / \delta}, X_{1}\right)$

Proof. Let $f \in X_{1-1 / \delta}$ and $u=B_{\alpha}(f)$. Let $W: \Omega \rightarrow \mathbb{R}, W(\mathbf{x})=e^{\lambda\|\mathbf{x}\|^{2}}$. The job is now to prove that there exists $\lambda>0$ and $A^{\prime}>0$ independent on $f$, such that

$$
|u(\mathbf{x})| \leq A W(\mathbf{x}) M(\mathbf{x}), \quad \forall \mathbf{x} \in \Omega
$$

where we denoted $A=A^{\prime}\|f\|_{X_{1-1 / \delta}}$. Actually we take on to prove $A W M \geq u$ only, as $A W M \geq$ $-u$ can be proved similarly. To achieve this, we again make use of the Comparison Principle.

One has:

$$
L_{\alpha}(M W)=M[-\Delta W+\mathbf{k} \cdot \nabla W+(\alpha+\nabla \cdot \mathbf{k}) W]+\nabla M \cdot(\mathbf{k} W-\nabla W)
$$

However, $\nabla W=2 \lambda \mathbf{x} W, \Delta W=\left(2 \lambda n+\lambda^{2}\|\mathbf{x}\|^{2}\right) W$, hence:

$$
\begin{aligned}
L_{\alpha}(M W) & =M W\left(-4 \lambda^{2}\|\mathbf{x}\|^{2}-\lambda n+2 \mathbf{k} \cdot \lambda \mathbf{x}+\alpha+\nabla \cdot \mathbf{k}\right) \\
& +2 \delta M^{1-1 / \delta} W\left(2 \lambda\|\mathbf{x}\|^{2}-\mathbf{k} \cdot \mathbf{x}\right)
\end{aligned}
$$

Let us take $\lambda=\lambda_{0}$ with $\lambda_{0}$ given in (23). We obtain

$$
2 \lambda\|\mathbf{x}\|^{2}-\mathbf{k} \cdot \mathbf{x} \geq 1
$$


From hypothesis (22) on $\alpha$ we obtain

$$
-4 \lambda^{2}\|\mathbf{x}\|^{2}-\lambda n+2 \lambda \mathbf{k} \cdot \mathbf{x}+\alpha+\nabla \cdot \mathbf{k} \geq 0 \quad \text { on } \Omega-\Omega_{1 / 2}
$$

which gives

$$
L_{\alpha}(M W) \geq 2 \delta W M^{1-1 / \delta}, \forall \mathbf{x} \in \Omega-\Omega_{1 / 2} .
$$

Next, as $f \in X_{1-1 / \delta}$, one gets

$$
-f(\mathbf{x}) \geq-\|f\|_{X_{1-1 / \delta}} M^{1-1 / \delta}(\mathbf{x}), \forall \mathbf{x} \in \Omega
$$

and invoking further on (64) leads to:

$$
A L_{\alpha}(M W)-f \geq\left[2 \delta W A-\|f\|_{X_{1-1 / \delta}}\right] M^{1-1 / \delta}, \forall \mathbf{x} \in \Omega-\Omega_{1 / 2}, \forall A>0 .
$$

Choose $A>0$ such that $2 \delta A W(\mathbf{x}) \geq\|f\|_{X_{1-1 / \delta}}, \forall \mathbf{x} \in \Omega-\Omega_{1 / 2}$. For instance, any $A$ such that:

$$
A \geq \frac{1}{2 \delta}\|f\|_{X_{1-1 / \delta}}
$$

will fit in. Then:

$$
L_{\alpha}(A M W-u) \geq 0 \quad \text { on } \quad \Omega-\Omega_{1 / 2}
$$

On the other hand, one needs to choose $A$ so that $A M W \geq u$ holds true over $\overline{\Omega_{1 / 2}}$. We proceed as in the proof of Lemma 3.4. A sound choice for $A$ is one such that

$$
A\left(\min _{\mathbf{x} \in \Omega_{1 / 2}} W(\mathbf{x})\right)\left(\min _{\mathbf{x} \in \Omega_{1 / 2}} M(\mathbf{x})\right) \geq \max _{\mathbf{x} \in \Omega} u(\mathbf{x})
$$

Next, $\min _{\mathbf{x} \in \Omega_{1 / 2}} W(\mathbf{x})=1, \min _{\mathbf{x} \in \Omega_{1 / 2}} M(\mathbf{x})=(3 / 4)^{\delta}$, and we are left over to inquire about $\max _{\mathbf{x} \in \Omega} u(\mathbf{x})$. To get an answer to, we shall call in Lemma 3.3 with $\beta_{1}=1-1 / \delta$ and $\beta_{2}=1 / 2-1 / \delta$. One has

$$
\|u\|_{\mathcal{C}(\bar{\Omega})} \leq c_{1}\|u\|_{X_{1 / 2-1 / \delta}} \leq c_{2}\|f\|_{X_{1-1 / \delta}}
$$


Then, one may choose $A \geq(4 / 3)^{\delta} c_{2}\|f\|_{X_{1-1 / \delta}}$ to ensure (68) holds true. Finally, taking into account (66), we are left to choose

$$
A=\max \left\{1 /(2 \delta),(4 / 3)^{\delta} c_{2}\right\}\|f\|_{X_{1-1 / \delta}}
$$

and we end the proof exactly as in Lemma 3.4, taking into account the fact that $M W \in H_{M}^{1}$, so that the Comparison Principle can be made use of.

\subsection{Strong Maximum Principle for the $B_{\alpha}$ operator}

This section aim is to prove the following "Strong Maximum Principle " property for $B_{\alpha}$ : for any $f \in P_{1}-\{0\}, B_{\alpha} f \in \stackrel{\circ}{P}$.

The following weaker result is first proved.

Lemma 3.6. Let $f \in P_{1}, f \neq 0$ and $u=B_{\alpha} f$. Then $u(\mathbf{x})>0, \forall \mathbf{x} \in \Omega$.

Proof. We adapt here the classical proof for the case where $M$ is equal to 1 (the non-degenerate case; see for example Gilbarg and Trudinger [12] or Evans [21]).

We remark first that $u$ is continuous on $\Omega$. Assume $\exists \mathbf{x} \in \Omega$ such that $u(\mathbf{x})=0$.

Denote $V_{e}:=\{\mathbf{x} \in \Omega, u(\mathbf{x})=0\}, V_{s}:=\{\mathbf{x} \in \Omega, u(\mathbf{x})>0\}, V_{e} \cup V_{s}=\Omega$.

By hypothesis $V_{e} \neq \emptyset$, as well as $V_{s} \neq \emptyset$. It is clear that $V_{s}$ is open and that $\partial V_{e} \nsubseteq \partial \Omega$. Let $\mathbf{z}_{0} \in \partial V_{e} \cap \Omega \neq \emptyset$; then $u\left(\mathbf{z}_{0}\right)=\mathbf{0}$. Denote $d=\inf _{\mathbf{z} \in \partial \Omega}\left|\mathbf{z}_{0}-\mathbf{z}\right|>0$, thus $\left|\mathbf{z}_{0}\right|=1-d$. Let $\left.r_{1} \in\right] 0, d / 4[$ be small enough, and fix $\mathbf{x}_{0} \in V_{s}$ such that $\left|\mathbf{x}_{0}-\mathbf{z}_{0}\right|<r_{1}$. As $V_{s}$ is an open subset, there exists $r_{2}>0$ such that $B\left(\mathbf{x}_{0}, r_{2}\right) \subset V_{s}$. Therefore choose $r_{0}=\sup \left\{r\right.$ s.t. $\left.B\left(\mathbf{x}_{0}, r\right) \subset V_{s}\right\}$. Then there exists $\mathbf{y}_{0} \in \overline{B\left(\mathbf{x}_{0}, r_{0}\right)} \cap V_{e} \neq \emptyset, u\left(\mathbf{y}_{0}\right)=0$, and $\left|\mathbf{y}_{0}-\mathbf{x}_{0}\right|=r_{0}$. This prompts $r_{0} \leq r_{1}$, hence one may choose a small enough $r_{0}$. Thus, $u\left(\mathbf{y}_{0}\right)=0, u(\mathbf{x})>0, \forall \mathbf{x} \in B\left(\mathbf{x}_{0}, r_{0}\right)$. Let the function $w$ be such that $w: B\left(\mathbf{x}_{0}, r_{0}\right) \rightarrow \mathbb{R}, w(\mathbf{x})=e^{-\lambda\left\|\mathbf{x}-\mathbf{x}_{0}\right\|^{2}}-e^{-\lambda r_{0}^{2}}$, where $\lambda>0$ will be 
later chosen conveniently. Denote also by $w$ the continuous extention of $w$ at 0 on $\Omega$ and note that $w \in H_{M}^{1}(\Omega),\left.w(\mathbf{x})\right|_{\mathbf{x} \in \partial B\left(\mathbf{x}_{0}, r_{0}\right)}=0$, and $\left.w(\mathbf{x})\right|_{\mathbf{x} \in B\left(\mathbf{x}_{0}, r_{0}\right)}>0$.

Next, we take on to prove that $\exists A>0$ such that $u(\mathbf{x}) \geq A w(\mathbf{x}), \forall \mathbf{x} \in B\left(\mathbf{x}_{0}, r_{0}\right)-$ $B\left(\mathbf{x}_{0}, r_{0} / 2\right)$. To achieve this we shall make use of the Comparison Principle. We actually evaluate $L_{\alpha}(u-A w)=f-A L_{\alpha}(w), f \geq 0$, and prove that $L_{\alpha}(w) \leq 0$ for any $\forall \mathbf{x} \in$ $B\left(\mathbf{x}_{0}, r_{0}\right)-B\left(\mathbf{x}_{0}, r_{0} / 2\right)$

Basic calculations lead to:

$$
L_{\alpha}(w)=E_{1}+E_{2}
$$

where, in the above

$$
\begin{aligned}
& E_{1}=-\Delta w+\left(\frac{\nabla M}{M}+\mathbf{k}\right) \cdot \nabla w \\
& E_{2}=\left[\nabla \cdot \frac{\nabla M}{M}+\nabla \cdot \mathbf{k}+\alpha\right] w=\left[\frac{\Delta M}{M}-\frac{|\nabla M|^{2}}{M^{2}}+\nabla \cdot \mathbf{k}+\alpha\right] w
\end{aligned}
$$

Using the expression of $w$ we find

$$
\begin{aligned}
& E_{1}=\left[-4 \lambda^{2}\left|\mathbf{x}-\mathbf{x}_{0}\right|^{2}+2 \lambda n+\frac{4 \delta \lambda \mathbf{x} \cdot\left(\mathbf{x}-\mathbf{x}_{0}\right)}{1-\|\mathbf{x}\|^{2}}\right. \\
& \left.-2 \lambda \mathbf{k} \cdot\left(\mathbf{x}-\mathbf{x}_{0}\right)\right] e^{-\lambda\left|\mathbf{x}-\mathbf{x}_{0}\right|^{2}}
\end{aligned}
$$

and

$$
\begin{aligned}
& E_{2}=\left[-\frac{2 n \delta}{1-\|\mathbf{x}\|^{2}}+\frac{4 \delta(\delta-1)\|\mathbf{x}\|^{2}}{\left(1-\|\mathbf{x}\|^{2}\right)^{2}}-\frac{4 \delta^{2}\|\mathbf{x}\|^{2}}{\left(1-\|\mathbf{x}\|^{2}\right)^{2}}\right. \\
& +\nabla \cdot \mathbf{k}+\alpha]\left(e^{-\lambda\left|\mathbf{x}-\mathbf{x}_{0}\right|^{2}}-e^{-\lambda r_{0}^{2}}\right)
\end{aligned}
$$

Next, observe that $\left\|\mathbf{x}_{0}\right\| \leq \| \leq 1-d+r_{1}$, so for any $\mathbf{x} \in B\left(\mathbf{x}_{0}, r_{0}\right)$, one has $\|\mathbf{x}\| \leq$ $1-d+r_{1}+r_{0} \leq 1-d / 2$. Therefore $1-\|\mathbf{x}\|^{2} \geq d(1-d / 4)>0$. Denote $d_{0}=1 /\left(d-d^{2} / 4\right)>0 ;$ hence

$$
\frac{1}{1-\|\mathbf{x}\|^{2}} \leq d_{0}, \quad \forall \mathbf{x} \in B\left(\mathbf{x}_{0}, r_{0}\right)
$$


Then

$$
E_{1} \leq\left[-\lambda^{2} r_{0}^{2}+2 \lambda n+4 \delta \lambda r_{0} d_{0}+2 \lambda\|\mathbf{k}\|_{L^{\infty}} r_{0}\right] e^{-\lambda\left|\mathbf{x}-\mathbf{x}_{0}\right|^{2}}, \quad \forall \mathbf{x} \text { such that } \frac{r_{0}}{2} \leq\|\mathbf{x}\| \leq r_{0} .
$$

We also have

$$
E_{2} \leq\left|E_{2}\right| \leq\left[2 n \delta d_{0}+4 \delta(\delta-1) d_{0}^{2}+4 \delta^{2} d_{0}^{2}+\|\nabla \cdot \mathbf{k}\|_{L^{\infty}}+\alpha\right] e^{-\lambda\left|\mathbf{x}-\mathbf{x}_{0}\right|^{2}}
$$

which implies

$$
\begin{aligned}
L_{\alpha}(w) \leq & {\left[-\lambda^{2} r_{0}^{2}+\lambda\left(2 n+4 \delta r_{0} d_{0}+2\|\mathbf{k}\|_{L^{\infty}} r_{0}\right)+2 n \delta d_{0}+\left(8 \delta^{2}-4 \delta\right) d_{0}^{2}+\|\nabla \cdot \mathbf{k}\|_{L^{\infty}}+\alpha\right] e^{-\lambda\left|\mathbf{x}-\mathbf{x}_{0}\right|^{2}}, } \\
& \forall \mathbf{x} \text { such that } \frac{r_{0}}{2} \leq\|\mathbf{x}\| \leq r_{0} .
\end{aligned}
$$

Then one may choose a $\lambda>0$ large enough (with $\lambda$ depending on $z_{0}$ and $r_{0}$ ) so that the rhs be negative, i.e. $L_{\alpha}(w) \leq 0$. Therefore

$$
L_{\alpha}(u-A w) \geq 0, \quad \forall \mathbf{x} \in B\left(\mathbf{x}_{0}, r_{0}\right)-B\left(\mathbf{x}_{0}, r_{0} / 2\right), \quad \forall A>0 .
$$

Next, as $u(\mathbf{x})>0$ in $B\left(\mathbf{x}_{0}, r_{0}\right)$ and $u$ is continuous in $\Omega$, one has $\inf _{\mathbf{x} \in \partial B\left(\mathbf{x}_{0}, r_{0} / 2\right)} u(\mathbf{x})>0$. Choose $A>0$ such that $\inf _{\mathbf{x} \in \partial B\left(\mathbf{x}_{0}, r_{0} / 2\right)} u(\mathbf{x}) \geq A \sup _{\mathbf{x} \in \partial B\left(\mathbf{x}_{0}, r_{0} / 2\right)} w(\mathbf{x})=A\left(e^{-\lambda r_{0}^{2} / 4}-e^{-\lambda r_{0}^{2}}\right)$. Then choose $A=\left[\inf _{\mathbf{x} \in \partial B\left(\mathbf{x}_{0}, r_{0} / 2\right)} u(\mathbf{x})\right] /\left[e^{-\lambda r_{0}^{2} / 4}-e^{-\lambda r_{0}^{2}}\right]$, to get

$$
u \geq A w \quad \text { on } \quad \partial B\left(\mathbf{x}_{0}, r_{0} / 2\right)
$$

We also have

$$
u \geq A w \quad \text { on } \quad \partial B\left(\mathbf{x}_{0}, r_{0}\right)
$$

Then the inequality (75) and the Comparison Principle give

$$
u \geq A w, \quad \forall \mathbf{x} \in B\left(\mathbf{x}_{0}, r_{0}\right)-B\left(\mathbf{x}_{0}, r_{0} / 2\right) .
$$

Next, the interior regularity property gives $u \in \mathcal{C}^{1}(\Omega)$. Let $\nu=\frac{\mathbf{y}_{0}-\mathbf{x}_{0}}{r_{0}}$ denote the outward normal vector at $\mathbf{y}_{0} \in B\left(\mathbf{x}_{0}, r_{0}\right)$. Then $\frac{\partial u}{\partial \nu}\left(\mathbf{y}_{0}\right)=-\frac{1}{r_{0}} \lim _{\substack{s \rightarrow 0 \\ s>0}} \frac{1}{s} u\left[\mathbf{y}_{0}-s\left(\mathbf{y}_{0}-\mathbf{x}_{0}\right)\right]$. 
With the help of inequality $((\sqrt{76}))$ it easily follows that:

$$
\frac{\partial u}{\partial \nu}\left(\mathbf{y}_{0}\right) \leq-2 \lambda A r_{0}^{2} e^{-\lambda r_{0}^{2}}<0
$$

On the other hand now, $\mathbf{y}_{0}$ is an interior point at which $u$ reaches a minimum $(u(\mathbf{x}) \geq 0$ on $\Omega$, $\left.u\left(\mathbf{y}_{0}\right)=0\right)$; this entails $\nabla u\left(\mathbf{y}_{0}\right)=0$, hence $\frac{\partial u}{\partial \nu}\left(\mathbf{y}_{0}\right)=0$, which contradicts inequality (77). This last argument ends the proof.

The main result of this section is

\section{Lemma 3.7.}

$$
B_{\alpha}\left(P_{1}-\{0\}\right) \subset \stackrel{\circ}{P_{1}}
$$

Proof. Since Lemma 3.5 gives $B_{\alpha}\left(X_{1}\right) \subset X_{1}$, it suffices to prove that for any $f \in P_{1}, f \not \equiv 0$, there exists $c>0$, such that

$$
u(\mathbf{x}) \geq c M(\mathbf{x}), \quad \forall \mathbf{x} \in \Omega
$$

where in the above $u=B_{\alpha}(f)$.

The difficulty here is to lower bound $u$ in a neighborhood of the boundary of $\Omega$.

Let $W_{0}: \Omega \rightarrow \mathbb{R}, W_{0}(\mathbf{x})=e^{-\lambda\|\mathbf{x}\|^{2}}-e^{-\lambda}$, with $\lambda>0$, and

$W_{1}: \Omega \rightarrow \mathbb{R}, W_{1}(\mathbf{x})=\left[W_{0}(\mathbf{x})\right]^{\delta}$. Observe that $W_{0}(\mathbf{x})=e^{-\lambda}\left[e^{\lambda\left(1-\|\mathbf{x}\|^{2}\right)}-1\right] ;$ using now the inequalities

$$
e^{z}-1 \geq z \quad \text { and } \quad e^{z}-1 \leq z e^{\lambda} \quad \text { with } \quad z=\lambda\left(1-\|x\|^{2}\right)
$$

we deduce

$$
\lambda e^{-\lambda}\left(1-\|\mathbf{x}\|^{2}\right) \leq\left|W_{0}(\mathbf{x})\right| \leq \lambda\left(1-\|\mathbf{x}\|^{2}\right), \quad \forall \mathbf{x} \in \Omega
$$

which implies

$$
\lambda^{\delta} e^{-\delta \lambda} M(\mathbf{x}) \leq\left|W_{1}(\mathbf{x})\right| \leq \lambda^{\delta} M(\mathbf{x}), \quad \forall \mathbf{x} \in \Omega
$$

Then $W_{1} \in X_{1}$ which triggers $W_{1} \in L_{M}^{2}$.

The followings hold true as well: $\nabla W_{0}=-2 \lambda \mathbf{x} e^{-\lambda\|\mathbf{x}\|^{2}}$ and $\nabla W_{1}=-2 \lambda \delta \mathbf{x} e^{-\lambda\|\mathbf{x}\|^{2}} W_{0}^{\delta-1}$. Inequality (79) leads to $\left|\nabla W_{1}\right| \leq c M^{1-1 / \delta}$. Finally, Proposition 3.4 gives $W_{1} \in H_{M}^{1}$. 
We take on to proving $L_{\alpha}\left(u-A W_{1}\right) \equiv f-A L_{\alpha}\left(W_{1}\right) \geq 0$ on $\Omega-\Omega_{\eta}$, where $A>0$ and $\eta \in] 0,1\left[\right.$ will be chosen later. As $f \geq 0$, we need to prove that $L_{\alpha}(W) \leq 0$. One has:

$$
L_{\alpha}\left(W_{1}\right)=-\Delta W+\left(\frac{\nabla M}{M}+\mathbf{k}\right) \cdot \nabla W+\left(\frac{\Delta M}{M}-\frac{|\nabla M|^{2}}{M^{2}}+\nabla \cdot \mathbf{k}+\alpha\right) W .
$$

Carrying out the calculations by making explicit $\nabla M, \Delta M$, etc, leads to

$$
\begin{aligned}
L_{\alpha}\left(W_{1}\right) & =2 \lambda \delta n e^{-\lambda\|\mathbf{x}\|^{2}} W_{0}^{\delta-1}-4 \lambda^{2} \delta\|\mathbf{x}\|^{2} e^{-\lambda\|\mathbf{x}\|^{2}} W_{0}^{\delta-1}-4 \lambda^{2} \delta(\delta-1)\|\mathbf{x}\|^{2} e^{-2 \lambda\|\mathbf{x}\|^{2}} W_{0}^{\delta-2} \\
& +4 \lambda \delta^{2} \frac{\|\mathbf{x}\|^{2}}{1-\|\mathbf{x}\|^{2}} e^{-\lambda\|\mathbf{x}\|^{2}} W_{0}^{\delta-1}-2 \lambda \delta \mathbf{k} \cdot \mathbf{x} e^{-\lambda\|\mathbf{x}\|^{2}} W_{0}^{\delta-1}-\frac{2 n \delta}{1-\|\mathbf{x}\|^{2}} W_{0}^{\delta} \\
& +4 \delta(\delta-1) \frac{\|\mathbf{x}\|^{2}}{\left(1-\|\mathbf{x}\|^{2}\right)^{2}} W_{0}^{\delta}-4 \delta^{2} \frac{\|\mathbf{x}\|^{2}}{1-\|\mathbf{x}\|^{2}} W_{0}^{\delta}+(\nabla \cdot \mathbf{k}+\alpha) W_{0}^{\delta}
\end{aligned}
$$

Denote now $y=1-\|\mathbf{x}\|^{2}$. Expanding about " $y$ close to 0 " leads to $W_{0}=\lambda e^{-\lambda} y\left(1+\frac{\lambda y}{2}+\frac{(\lambda y)^{2}}{6} e^{z_{1}}\right)$ with $z_{1} \in[0, \lambda]$.

We then have, for any $\gamma>0$,

$$
W_{0}^{\gamma}=\lambda^{\gamma} e^{-\lambda \gamma} y^{\gamma}\left(1+\frac{\lambda \gamma y}{2}+y^{2} h(y, \lambda, \gamma)\right)
$$

where, due to the fact that $y \in[0,1], h$ is such that

$$
|h(y, \lambda, \gamma)| \leq \bar{h}
$$

where $\bar{h}$ is a positive constant depending on $\lambda$ and $\gamma$. Next, for any $\gamma>0$,

$$
e^{-\gamma \lambda\|\mathbf{x}\|^{2}}=e^{-\gamma \lambda} e^{\gamma \lambda y}=e^{-\gamma \lambda}\left(1+\gamma \lambda y+\frac{(\gamma \lambda y)^{2}}{2} e^{z_{2}}\right)
$$

with $z_{2} \in[0, \lambda \gamma]$.

Expand the right-hand side of (80) in power series w.r.t. $y$. Using (81) and (82) and taking into account the equality $\|x\|^{2}=1-y$, one remarks that the coefficients of the leading term $y^{\delta-2}$ vanish, so after some lengthy (and awkward) algebra one gets:

$$
L_{\alpha}\left(W_{1}\right)=a_{1}(\mathbf{x}, \lambda) y^{\delta-1}+a_{2}(\mathbf{x}, y, \lambda) y^{\delta}
$$

with

$$
a_{1}(\mathbf{x}, \lambda)=2 \lambda^{\delta} e^{-\delta \lambda}\left(-\delta^{2} \lambda+4 \delta^{2}-\delta \mathbf{x} \cdot \mathbf{k}\right)
$$


and $a_{2}$ a function satisfying

$$
\left|a_{2}(\mathbf{x}, y, \lambda)\right| \leq \bar{a}_{2}
$$

where $\bar{a}_{2}$ is a positive constant depending in $\lambda$. Next,

$-\delta^{2} \lambda+4 \delta^{2}-\delta \mathbf{x} \cdot \mathbf{k} \leq-\delta^{2} \lambda+4 \delta^{2}+\delta\|\mathbf{k}\|_{L^{\infty}}$ and with a suitable choice for $\lambda$, such as:

$$
\lambda=\frac{1}{\delta^{2}}\left(4 \delta^{2}+\delta\|\mathbf{k}\|_{L^{\infty}}+1\right)
$$

one gets $4 \delta^{2}-\delta^{2} \lambda-\delta \mathbf{x} \cdot \mathbf{k} \leq-1$, which gives

$$
a_{1}(\mathbf{x}, \lambda) \leq-2 \lambda^{\delta} e^{-\delta \lambda}
$$

Therefore

$$
L_{\alpha}\left(W_{1}\right) \leq-2 \lambda^{\delta} e^{-\delta \lambda} y^{\delta-1}+\bar{a}_{2} y^{\delta}=-2 \lambda^{\delta} e^{-\delta \lambda} y^{\delta-1}\left(1-\frac{\bar{a}_{2}}{2} \lambda^{-\delta} e^{\delta \lambda} y\right)
$$

Then one may take $y$ small enough (i.e. $\|\mathbf{x}\|$ close to 1 ) such that $L_{\alpha}\left(W_{1}\right) \leq 0$.

It has thus been proved that $\exists \eta \in] 0,1[$, close to 1 , such that

$$
L_{\alpha}\left(u-A W_{1}\right) \geq 0, \quad \forall \mathbf{x} \in \Omega-\Omega_{\eta}, \quad \forall A>0 .
$$

Next, from Lemma 3.6 we have $u>0$ over $\Omega$. Since $u$ is also continuous, $A$ may be chosen such that $\min _{\mathbf{x} \in \bar{\Omega}_{\eta}} u(\mathbf{x}) \geq A \underbrace{A \max _{\mathbf{x} \in \bar{\Omega}_{\eta}} W(\mathbf{x})}_{\leq 2^{\delta}}$. Take $A=\frac{1}{2^{\delta}} \min _{\mathbf{x} \in \bar{\Omega}_{\eta}} u(\mathbf{x})$. Such a choice leads to

$$
u(\mathbf{x}) \geq A W_{1}(\mathbf{x}), \forall \mathbf{x} \in \bar{\Omega}_{\eta}
$$

Since $u, W_{1} \in H_{M}^{1}$, use of equations (88), (89) and of Comparison Principle allows one to infer that $u(\mathbf{x}) \geq A W_{1}(\mathbf{x}), \forall \mathbf{x} \in \Omega-\Omega_{\eta}$. One more use of (89) implies that, in fact, this inequality holds true on the entire $\Omega$ domain. Now the inequality (79) gives the result. 


\section{Proof of the main result}

We are now in position to give the proof of Theorem 2.1.

\section{Step 1.}

From part (ii) in Lemma 3.3 one infers

$$
B_{\alpha} \in \mathscr{L}\left(X_{1}, X_{1 / 2-1 / \delta}\right)
$$

From Lemma 3.4 we obtain

$$
B_{\alpha} \in \mathscr{L}\left(X_{1 / 2+(2 j-1) / \delta}, X_{1 / 2+(2 j+1) / \delta}\right), \quad \text { for any } j \in\left\{0,1, \cdots j_{0}\right\}
$$

where

$$
j_{0}=\max \left\{j \in \mathbb{N}, \quad \frac{1}{2}+\frac{2 j+1}{\delta}<1\right\}
$$

(in other words, $j_{0}$ is the unique natural number belonging to the interval $\left[\frac{\delta}{4}-\frac{3}{2}, \frac{\delta}{4}-\frac{1}{2}[\right.$ ).

Due to the inequality $\frac{1}{2}+\frac{2 j_{0}+1}{\delta} \geq 1-\frac{2}{\delta}$ we have the inclusion $X_{1 / 2+\left(2 j_{0}+1\right) / \delta} \subset X_{1-3 / \delta}$. Using again Lemma 3.4 we obtain

$$
B_{\alpha}^{j_{0}+2} \in \mathscr{L}\left(X_{1}, X_{1-1 / \delta}\right)
$$

Finally, from Lemma 3.5 we deduce

$$
S_{\alpha} \in \mathscr{L}\left(X_{1}, X_{1}\right)
$$

where the following notation has been used:

$$
S_{\alpha}=B_{\alpha}^{j_{0}+3}
$$

As $B_{\alpha}$ is a compact operator that maps $X_{1}$ onto $X_{1 / 2-1 / \delta}$ (see part (ii) in Lemma 3.3) it follows that $S_{\alpha}$ is compact as well.

On the other hand, Lemma 3.7 gives

$$
S_{\alpha}\left(P_{1}-\{0\}\right) \subset \stackrel{\circ}{P}_{1}
$$


We are now in a position that allows to make use of the strong version of the Krein-Rutman theorem (see for example [22]) to the operator $S_{\alpha}$, the Banach space $X_{1}$ and the cone $P_{1}$. One deduces the existence of an eigenvalue $\mu_{0}>0$ of $S_{\alpha}$, to which corresponds the eigenvector $u_{0} \in \stackrel{\circ}{P}_{1}$, that is

$$
B_{\alpha}^{j_{0}+3} u_{0}=\mu_{0} u_{0}
$$

Moreover, if $u_{1} \in \stackrel{\circ}{P}_{1}$ is any other eigenvector of $S_{\alpha}$, related to a positive eigenvalue, then $u_{1}$ is equal to $u_{0}$ up to a multiplicative positive constant.

\section{Step 2 (Existence).}

Denote by $\operatorname{Spr}\left(B_{\alpha}\right)$ the spectral radius of $B_{\alpha}$, where $B_{\alpha}$ is considered an element of $\mathscr{L}\left(L_{M}^{2}\right)$. It is well known (see for example Section III.6.2 in [23]) that

$$
\operatorname{Spr}\left(B_{\alpha}\right)=\lim _{m \rightarrow+\infty}\left\|B_{\alpha}^{m}\right\|_{\mathscr{L}\left(L_{M}^{2}\right)}^{1 / m}
$$

Since $u_{0} \in L_{M}^{2}$ and $u_{0} \neq 0$, using $\left.(91)\right)$ one has:

$$
\left\|B_{\alpha}^{m\left(j_{0}+3\right)}\right\|_{\mathscr{L}\left(L_{M}^{2}\right)} \geq \frac{\left\|B_{\alpha}^{m\left(j_{0}+3\right)} u_{0}\right\|_{L_{M}^{2}}}{\left\|u_{0}\right\|_{L_{M}^{2}}}=\mu_{0}^{m}
$$

which triggers

$$
\operatorname{Spr}\left(B_{\alpha}\right) \geq \mu_{0}^{1 /\left(j_{0}+3\right)}>0
$$

On the other hand, let us denote by $P_{M}^{2}$ the (reproducible) cone of positive functions in $L_{M}^{2}$. Due to the Weak Maximum Principle (Lemma 3.1),

$$
B_{\alpha}\left(P_{M}^{2}\right) \subset P_{M}^{2}
$$

We also have that $B_{\alpha}$ is a compact operator from $L_{M}^{2}$ onto itself (due to the compact embedding of $H_{M}^{1}$ in $L_{M}^{2}$, see [24]).

Use now the weak version of the Krein-Rutman theorem (see [22]) for the operator $B_{\alpha}$, the Banach space $L_{M}^{2}$ and the cone $P_{M}^{2}$. It turns out there exists an eigenvalue $\tilde{\mu}_{0}>0$ of $B_{\alpha}$ and a corresponding eigenvector $\tilde{u}_{0} \in P_{M}^{2}-\{0\}$, i.e.

$$
B_{\alpha} \tilde{u}_{0}=\tilde{\mu}_{0} \tilde{u}_{0} .
$$


Clearly $\tilde{u}_{0}$ also belongs to $H_{M}^{1}$. Moreover, any other eigenvalue $\mu \in \mathbb{C}$ of $B_{\alpha}$ is such that

$$
|\mu| \leq \tilde{\mu}_{0}
$$

Let us now denote $\tilde{\lambda}_{0}=1 / \tilde{\mu}_{0}-\alpha ; \tilde{\lambda}_{0}$ is clearly an eigenvalue of $L$ related to the same eigenfunction $\tilde{u}_{0}$. It then follows:

$$
\int_{\Omega} M \nabla\left(\frac{\tilde{u}_{0}}{M}\right) \cdot \nabla\left(\frac{\varphi}{M}\right) \mathrm{d} \mathbf{x}-\int_{\Omega} \mathbf{k} \tilde{u}_{0} \cdot \nabla\left(\frac{\varphi}{M}\right) \mathrm{d} \mathbf{x}=\tilde{\lambda}_{0} \int_{\Omega} \frac{\tilde{u}_{0} \varphi}{M} \mathrm{~d} \mathbf{x}, \quad \forall \varphi \in H_{M}^{1} .
$$

Set $\varphi=M$ in the above equation, and since $\int_{\Omega} \tilde{u}_{0}>0\left(\right.$ remark that $\tilde{u}_{0} \in L^{1}(\Omega)$ by the obvious embedding $\left.L_{M}^{2}(\Omega) \underset{\text { cont }}{\subset} L^{1}(\Omega)\right)$, we deduce that $\tilde{\lambda}_{0}=0$. Then the following expression:

$$
\psi=\frac{b}{\int_{\Omega} \tilde{u}_{0} \mathrm{~d} \mathbf{x}} \tilde{u}_{0}
$$

gives a solution of eq(17a).

Step 3 (Uniqueness).

Assume $\psi_{1}$ and $\psi_{2}$ are two solutions to the problem $(17 \mathrm{a})-(17 \mathrm{~g})$. Then $\psi_{1}$ and $\psi_{2}$ are nonnegative eigenvectors of operator $L$ corresponding to the eigenvalue 0 . This implies

$$
B_{\alpha} \psi_{m}=\frac{1}{\alpha} \psi_{m}, \quad m=1,2
$$

which gives

$$
S_{\alpha} \psi_{m}=\left(\frac{1}{\alpha}\right)^{j_{0}+3} \psi_{m}, \quad m=1,2 .
$$

We then obtain $\psi_{m} \in X_{1}$ and by $((90))$ we also have $\psi_{m} \in \stackrel{\circ}{P}_{1}, m=1,2$. Now by the uniqueness property of the strong version of the Krein-Rutman theorem, there exists $r>0$ such that $\psi_{1}(\mathbf{x})=r \psi_{2}(\mathbf{x}), \quad \forall \mathbf{x} \in \Omega$. Now since $\int_{\Omega} \psi_{1}=\int_{\Omega} \psi_{2}=b$ we obtain $r=1$ which proves the uniqueness. 
Remark 4.1. From inequality ((93)) one can deduce, proceeding in a classical manner, that

$$
\operatorname{Re}(\lambda) \geq 0
$$

for any other complex eigenvalue of operator L. The eigenvalue 0 is then the principal eigenvalue of the operator $L$. Moreover, what is quite remarkable is the fact that 0 is the principal eigenvalue of $L$ for any function $\mathbf{k} \in\left(W^{1, \infty}(\Omega)\right)^{n}$.

\section{$5 \quad$ Final comments}

We have offered a proof to the fact that the FENE dumbbell configurational distribution function diffusion equation - see the corresponding boundary value problem described in eqs (10a)(10d)- has unique steady state solutions. In doing so, we relied on the Krein - Rutman theory of elliptic operators.

There are several motivations for this work. In [1] asymptotic solutions for the probability density diffusion equation - valid for slow flows - are presented, but no proof for the existence of such solutions is offered. While in this work we have proved the existence of solutions to the diffusion equation for slow and fast flows (that is irrespective of whether the velocity gradient is "small" or "large"), the questions related to the convergence of explicit expansion solutions given in [1] (and in what functional space it occurs) are still to be addressed to the fullest. Moreover, we expect our results to further the work in finding asymptotic solutions valid for "large" velocity gradients, i.e. for fast flows, for the FENE dumbbell model; a solution in such a case is known only for rigid dumbbells (see [25]).

Now, the elastic (or rigid) dumbbell polymer chain models are certainly crude representations of the real chains. That set aside, they do capture several - but not all - important features of viscoelastic flows (e.g. shear rate dependent viscosity, first normal stress difference). Moreover, they owe a certain popularity among polymer scientists (from experimentalists to 
applied mathematicians) to their relative simplicity. More realistic models use different chain representations. For instance, Doi and Edwards [26] developed the so-called tube model for melt systems, that makes use of the de Genne's reptation ideas of anisotropic chain diffusion. Schweitzer and co-workers [27] developed a mode-coupling model in which the intermolecular structural constraints upon the motion of single macromolecules are modeled as a many body caging effect. Ngai and Plazek developed their own coupling model [28, 29], very successful in predicting the thermo-rheological complexity. This being said, bead-spring or bead-rod chain models still attract significant attention: see for example [30, 31, 32, 33, 34, 35]. For sure their full capabilities are still to be uncovered.

\section{Acknowledgements}

The authors thank Dr Jean-Francois Palierne, Laboratoire de Physique, Ecole Normale Supérieure de Lyon, and Dr Vitaly Volpert, Directeur de Recherche au CNRS, Université Lyon-I, for useful talks on polymer molecular dynamics, and on elliptic operators, respectively.

\section{References}

[1] R. B. Bird, R. C. Armstrong, O. Hassager, Dynamics of Polymeric Liquids, Vol. 2: Kinetic Theories, J. Wiley \& Sons, New-York, 1987.

[2] J. G. Kirkwood, Macromolecules, edited by P. L. Auer, Gordon and Breach, New York, 1968.

[3] H. R. Warner, Kinetic Theory and Rheology of Dilute Suspensions of Finitely Extendible Dumbbells, Ind. Eng. Chem. Fundam. 11 (3) (1972) 379 - 387. 
[4] M. D. Chilcott, J. M. Rallison, Creeping flow of dilute polymer solutions past cylinders and spheres, J. Non-Newtonian Fluid Mech. 29 (1988) 381 - 432.

[5] M. Herrchen, H. C. Öttinger, A detailed comparison of various FENE dumbbell models, J. Non-Newtonian Fluid Mech. 68 (1) (1997) 17 - 42.

[6] R. G. Larson, Constitutive Equations for Polymer Melts and Solutions, Butterworths, 1988.

[7] A. N. Beris, B. J. Edwards, Thermodynamics of Flowing Systems With Internal Microstructure, Oxford University Press, 1994.

[8] R. R. Huilgol, N. Phan-Thien, Fluid Mechanics of Viscoelasticity, Elsevier, 1997.

[9] F. A. Morrison, Understanding Rheology, Oxford University Press, 2001.

[10] H. C. Öttinger, Beyond Equilibrium Thermodynamics, Wiley, 2006.

[11] R. B. Bird, R. C. Armstrong, O. Hassager, Dynamics of Polymeric Liquids, Vol. 1: Fluid Mechanics, J. Wiley \& Sons, New-York, 1987.

[12] G. Gilbarg, N. Trudinger, Elliptic partial differential equations of second order, SpringerVerlag, Berlin, 2001.

[13] I. Ciuperca, M. El Alaoui, M. Jai, On the optimal control of coefficients in elliptic problems. Applications to the optimization of the head slides, ESAIM Control Optim. Calc. Var. 11(1) (2005) $101-121$.

[14] P. Degond, M. Lemou, M. Picasso, Viscoelastic fluid models derived from kinetic equations for polymers, SIAM J. Appl. Math. 62(5) (2002) 1501 - 1519.

[15] H. Zhang, P. Zhang, Local existence for the FENE-dumbbell model of polymeric fluids, Arch. Ratl. Mech. Anal. 181 (2006) 373 - 400. 
[16] F. Lin, P. Zhang, Z. Zhang, On the global existence of smooth solution to the 2-D FENE Dumbell Model, Commun. Math. Phys. 277 (2008) 531-553

[17] J. W. Barret, C. Schwab, E. Süli, Existence of global weak solutions for some polymeric flow models, Math. Model. Meth. Appl. Sci. 15(6) (2005) 939 - 983.

[18] B. Jourdain, T. Lelièvre, C. Le Bris, Existence of solution for a micro-macro model of polymeric fluid: the FENE model, Journal of Functional Analysis 209 (2004) 162-193.

[19] Q. Du, C. Liu, P. Yu, FENE dumbbell model and its several linear and nonlinear closure approximations, Multiscale Model. Simul. 4(3) (2005) 709 - 731.

[20] H. Triebel, Interpolation Theory, Function Spaces, Differential Operators, 2nd edition, Johann Ambrosius Barth Verlag, 1995.

[21] L.C. Evans, Partial Differential Equations, AMS, Providence, RI, 1998.

[22] R. Dautray, J.L. Lions, Analyse mathématique et calcul numérique pour les sciences et les techniques, Masson, Paris, 1964.

[23] T. Kato, Perturbation theory for linear operators, New York, 1966.

[24] J. Nečas, Les méthodes diréctes en théorie des équations elliptiques, Masson, Paris, 1967.

[25] H. C. Öttinger, A note on rigid dumbbell solutions at high shear rates, J. Rheol. 32(2) (1988) $135-143$.

[26] M. Doi, S. F. Edwards, The theory of polymer dynamics, 3rd edition, Oxford, 1989.

[27] K. S. Schwartz, M. Fuchs, G. Szamel, M. Guenza, H. Tang, Polymer-mode-coupling theory of the slow dynamics of entangled macromolecular fluids, Macromol. Theory Simul. 6 (1997) $1037-1117$. 
[28] K. L. Ngai, D. J. Plazek, Identification of different modes of molecular motion in polymers that cause thermorheological complexity, Rubber Chem. Technol. 68 (1995) 376 - 434.

[29] K. L. Ngai, Dynamic and thermodynamic properties of glass-forming substances, J. NonCryst. Solids 275 (2000) 7 - 51.

[30] C. Schneggenburger, M. Kröger, S. Hess, An extended FENE dumbbell theory for concentration dependent shear-induced anisotropy in dilute polymer solutions, J. Non-Newtonian Fluid Mech. 62 (2-3) (1996) 235 - 251.

[31] R. Sizaire, G. Lielens, I. Jaumain, R. Keunings, V. Legat, On the hysteretic behaviour of dilute polymer solutions in relaxation following extensional flow, J. Non-Newtonian Fluid Mech. 82 (2-3) (1999) 233 - 253.

[32] I. Ghosh, G. H. McKinley, R. A. Brown, R. C. Armstrong, Deficiencies of FENE dumbbell models in describing the rapid stretching of dilute polymer solutions, J. Rheol. 45 (3) (2001) $721-758$.

[33] J. G. Hernandez Cifre, Th. M. A. O. M. Barenbrug, J. D. Schieber, B. H. A. A. van den Brule, Brownian dynamics simulation of reversible polymer networks under shear using a non-interacting dumbbell model, J. Non-Newtonian Fluid Mech. 113 (2-3) (2003) 73 - 96.

[34] P. T. Underhill, P. S. Doyle, On the coarse-graining of polymers into bead-spring chains, J. Non-Newtonian Fluid Mech. 122 (1-3) (2004) 3 - 31.

[35] M. Kröger, E. De Angelis, An extended FENE dumbbell model theory for concentration dependent shear-induced anisotropy in dilute polymer solutions: addenda, J. Non-Newtonian Fluid Mech. 125 (1) (2005) 87 - 90. 